\title{
Improved Methodologies for Maneuver Design of Aircraft Stability and Control Simulations
}

\author{
Adam Jirásek*, Tiger L. Jeans†, Matthew Martenson; \\ Russell M. Cummings ${ }^{\S}$ and Keith Bergeron $₫$ \\ United States Air Force Academy, USAFA, CO 80840, USA
}

\begin{abstract}
With many modern fighter aircraft experiencing unpredicted flight dynamics during flight tests, recent research has focused on developing methodologies for incorporating computational fluid dynamics into the aircraft development process. The goal of this approach is to identify configurations susceptible to stability and control issues early in the design process. Previous research has primarily focused on full aircraft configurations, however, to increase the rate of development the current study focused on a two-dimensional NACA0012 airfoil. The two-dimensional NACA0012 airfoil has the advantage of reducing the computational cost by orders of magnitude compared to full scale aircraft simulations, while still providing complicated aerodynamics at high angles of attack. Computationally predicted lift coefficients from a number of newly developed training maneuvers were used to generate reduced order aerodynamic loads models. For evaluation, these models were compared to generated static and dynamic validation data. Methods of improving both the computational training maneuver and the reduced order modeling approach are suggested.
\end{abstract}

\section{Nomenclature}

speed of sound angle of attack induced angle of flow aerodynamic chord drag coefficient ( Lift $\left./ q_{\infty} S_{\text {ref }}\right)$ lift coefficient $\left(\mathrm{Drag} / q_{\infty} S_{\text {ref }}\right)$ pitching moment coefficient $\left(M_{z} / q_{\infty} S_{r e f} c\right)$ frequency of sinusoidal motion Mach number $v / a$ fluidic dynamic viscosity

Reynolds number $v_{\infty} c \rho_{\infty} / \mu_{\infty}$ density

time computational time step, seconds dimensionless computational time step free-stream speed of flow position

\section{Introduction}

$\mathrm{D}$ EFINING the Stability and Control (S\&C) characteristics of an airplane is probably one of the most difficult and expensive aspects of aircraft development. This is partially due to the fact that the S\&C

\footnotetext{
* National Research Council, NRC, Research Associate, Department of Aeronautics, AIAA Senior Member

$\dagger$ Visiting Researcher, Department of Aeronautics, Member AIAA

${ }^{\ddagger}$ Cadet First Class, Department of Aeronautics

$\S$ Professor of Aeronautics, Department of Aeronautics, AIAA Associate Fellow

`Director M\&SRC, Department of Aeronautics, Member AIAA
} 
design phase extends well into the development process, and sometimes even beyond, often resulting in unexpected aerodynamic issues being identified late in the development process. This can lead to costly adhoc fixes that are detrimental to the aircraft performance. Some recent examples include aircraft such as the F/A-18 and F-18E or F-22. ${ }^{1-6}$ It is therefore of utmost importance to be able to predict S\&C characteristics of the aircraft in the early stages of its development.

Several tools can be used to predict the S\&C characteristics of an aircraft, including flight and wind tunnel testing, semi-empirical lower-order modeling and computational fluid dynamics (CFD). Flight testing is the most accurate of these methods, but is also the most expensive and cannot be used during early stages of the aircraft development process because the aircraft configuration may not be finalized. Wind tunnel testing is also accurate, but suffers from scaling issues, along with difficulty modeling unsteady dynamic behavior. Wind tunnel testing is also expensive, although cheaper than flight testing. Semi-empirical lower-order modeling has less fidelity than flight and wind-tunnel testing and is incapable of reliably predicting unsteady nonlinear aerodynamic behavior. A reasonable compromise between flight and wind tunnel testing and semiempirical lower-order modeling is CFD simulation. Modern CFD techniques have a relatively high level of fidelity and have successfully modeled the non-linear aerodynamic behavior of aircraft at full scale Reynolds numbers. This method reduces some of the major uncertainties associated with sufficiently modeling physical space. However, it comes with an additional cost in execution time that results from computer performance and small physical time step requirements to accurately capture the flow physics. This is exaggerated by the low frequency nature of most of the aerodynamic motions that result in nonlinear behavior of interest. Researchers at NASA Ames, for example, have attempted to perform a "brute force" approach to filling a stability and control database for vehicle design. ${ }^{7-9}$ They found that a reasonable database for static stability and control derivatives would include on the order of 30 different angles-of-attack, 20 different Mach numbers, and 5 different side-slip angles, each for a number of different geometry configurations or control surface deflections. ${ }^{7}$ They envisioned that a few hundred solutions can be obtained automatically and the remainder of the parameter space is filled using an interpolation procedure or neural networks. Considering today's performance of computers and CFD codes, the routine calculations of hundreds of maneuvers in a reasonable time frame is unrealistic. In order to accurately and reliably predict the stability and control (S\&C) characteristics of an aircraft prior to the costly flight test phase, CFD has to be combined with a predictive modeling of lower complexity.

In response to this need, recent research at the United States Air Force Academy (USAFA) has focused on the application of CFD to aircraft S\&C, in particular, on how to effectively incorporate CFD into the S\&C model development process. The final goal is to develop a methodology for efficient and accurate screening of nonlinear aerodynamic phenomena such as spin, tumble, lateral instabilities, limit-cycle oscillations, and tail buffet of full scale aircraft early in the design process. To date, we have adopted the innovate approach introduced by McDaniel et al ${ }^{10}$ which is graphically represented in figure 1 , with a summary as follows: (a) CFD simulations are performed using computational training maneuvers designed to excite the relevant flow physics encountered during actual missions, (b) a mathematical Reduced Order Model (ROM) is built of the aircraft response using system identification methods, (c) the model is validated by comparing CFD simulations against model predictions, and (d) predictions of all flight test points are made using the model to determine the expected behavior of the aircraft.

The choice of both the computational training maneuver and the ROM approach is critical to the success of the above methodology. Currently, improvements are necessary to both for the computational tool to reach its full potential. Matthews and Schwartz, ${ }^{11}$ for example, showed that determining the appropriate training maneuver that properly excites all the relevant flow physics is not a trivial task but is vital to the success of the final aerodynamic model. In addition, previous research has primarily implemented SIDPAC ${ }^{12}$ as the ROM. SIDPAC is a nonlinear parameter modeling technique based on regression methods developed by Morelli specifically for aircraft applications. However, Jeans ${ }^{13}$ showed that this method is incapable of generating an aerodynamic loads model capable of predicting both nonlinear and linear aerodynamic behavior within a single training maneuver. In response to the above requirements, the primary objectives of this paper are twofold. First, to further investigate the appropriate computational training maneuver the efficiently probes the design space such that the ROM can interpolate to all possible combinations of the independent variables within the regressor space while still exciting the relevant flow physics, and second, to investigate a new ROM approach with better predictive capabilities then a least square approximation.

Previous simulations have primarily focused on three-dimensional and often full scale simulations of complete aircraft configurations. ${ }^{10,11,13-21}$ This was partially to validate that computational techniques have 


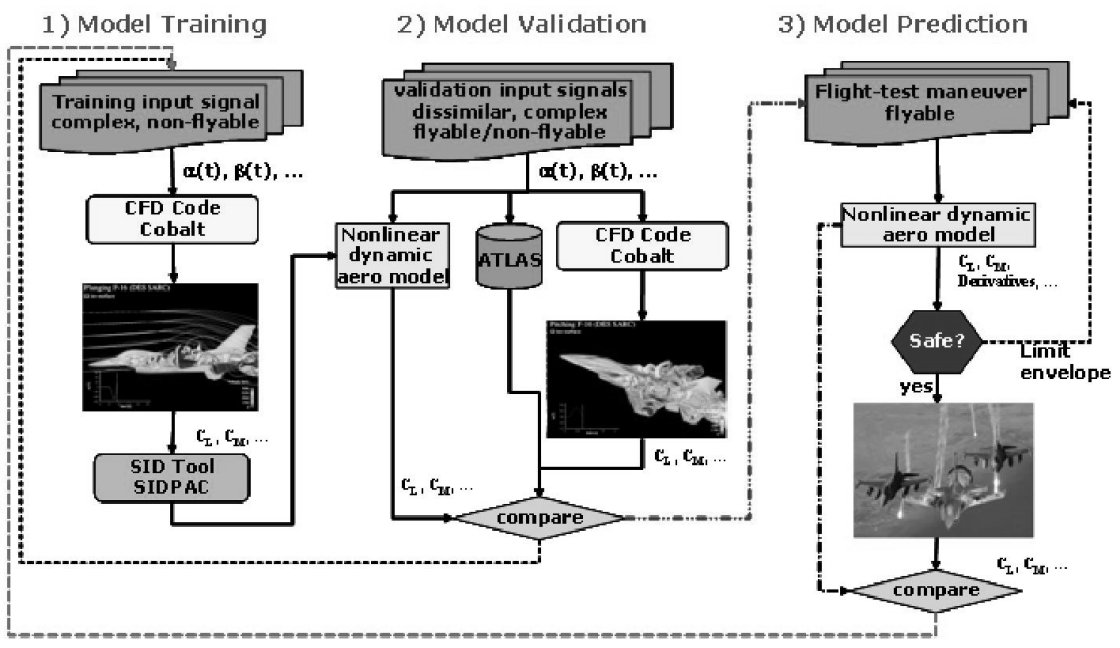

Figure 1. Stability and control model build process. ${ }^{10}$

progressed to the point where turbulent Navier-Stokes solvers are now capable of capturing the unsteady nonlinear aerodynamic behavior that leads to the various static and dynamic instabilities mentioned above. However, the cost of this approach was computationally expensive CFD simulations with a slow turn-around time, which severely hindered development. Given the rich history that currently exists for predicting massively separated high angle of attack flow using CFD, it was decided to simplify the geometry for the current study to two-dimensional airfoils. This approach drastically decreases the turn-around time for the various computational training maneuvers, while still allowing one to verify the methodologies for both linear and nonlinear aerodynamic regions by validating at high angles of attack that include stall. The premise being that any improvements made for a two-dimensional airfoil will also be valid for full aircraft configurations.

\section{S\&C Training Maneuvers}

The goal of a training maneuver is to produce enough representative data to build a reliable ROM with predictive capabilities within a desired regressor space. In addition, a systematic approach must be developed for determining how well a prospective maneuver meets this objective. Previous studies focused on training maneuver development, (see Ref ${ }^{12,14,22,23,23,24}$ ), used the frequency content and power density spectra characteristics of a particular maneuver as such a measure. Though correct for some applications, the same methodology cannot be used to characterize an appropriate maneuver used to predict S\&C characteristics of the aircraft. This is partially due to the fact that the motion of the aircraft is not primarily given in terms of frequency.

The basic requirement for a training maneuver that can be used to generate a reliable ROM in S\&C applications is that it sufficiently covers the desired regressor space of state variables. A ROM built on data produced by such motions can then be used to predict the aircraft's aerodynamic behavior within the regressor space if it is sufficiently covered. The systematic coverage of the regressor space can be, in general, treated as an optimization problem of filling the multidimensional space with strong constraints resulting from the fact that some axes of the regressor space do not represent an independent variable.

For the current study we primarily focused on one degree of freedom (DOF) motion, and in particular, a pitching and plunging airfoil. For this simple one DOF motion the regressor space is defined by the angle of attack, $\alpha$, and its first and second derivatives, $\dot{\alpha}$ and $\ddot{\alpha}$. The regressor space is covered using either a basic frequency or amplitude sweep. 


\section{A. Rotational motion}

\section{Chirp maneuver}

The chirp is an example of a frequency sweep with a constant amplitude and linearly increasing frequency. The angle of attack is specified in time as

$$
\alpha(t)=A \sin \left(\omega t^{2}\right)
$$

where $A$ is the amplitude of oscillations. The regressor space is symmetric about the initial angle of attack. Figure 2 shows the time history of $\alpha$ and regressor space coverage for two chirps with different amplitudes. Notice that the regressor coverage has very limited data in the low frequency region where most nonlinear aerodynamic behavior occurs. Altering the frequency growth rate of a linear chirp alters the density of the regressor space coverage shown in figure 2(b), but does not alter the overall shape of the coverage.

The DC chirp is a chirp with an initial offset and is defined as

$$
\alpha(t)=\frac{A}{2}\left(1-\cos \left(\omega t^{2}\right)\right) .
$$

Figure 3 shows two DC chirps in the time domain and their respective regressor space coverage. The DC chirp coverage of the regressor space is identical to the chirp, but with an offset in $\alpha_{0}$, the initial angle of attack.



(a) $t-\alpha$

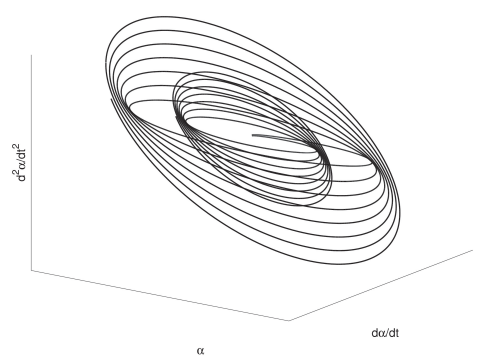

(b) $\alpha-\dot{\alpha}-\ddot{\alpha}$

Figure 2. Angle of attack as a function of time and regressor space coverage for the chirp

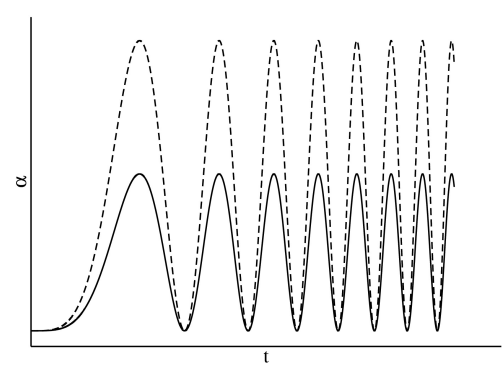

(a) $t-\alpha$



(b) $\alpha-\dot{\alpha}-\ddot{\alpha}$

Figure 3. Angle of attack as a function of time and regressor space coverage for the DC chirp maneuver

\section{Spiral maneuver}

Spiral maneuvers are amplitude sweeps with constant frequency. The amplitude increases linearly in time and the angle of attack as a function of time is

$$
\alpha(t)=A t \sin (\omega t),
$$

\section{4 of 27}


where $A$ is the rate of amplitude increase. Figure 4 shows the regressor map coverage of two spiral maneuvers with different frequencies. As can be seen in the figure the spiral maneuvers are so named due to the spiral nature of the regressor map coverage. The systematic coverage of the regressor space is then achieved by using several spirals with different frequency. Similar to the chirp maneuver, changing the amplitude growth rate alters the density of the regressor space coverage shown in figure 4(b), but does not alter the overall shape of the coverage.

The DC spiral is essentially a spiral with an initial offset and is defined as

$$
\alpha(t)=\frac{A}{2} t(1-\cos (\omega t))
$$

Figure 5 shows the angle of attack as a function of time and the resulting regressor space coverage for two DC spirals with different frequencies. Like the DC chirp, the DC spiral is not symmetric about $\alpha_{0}$.

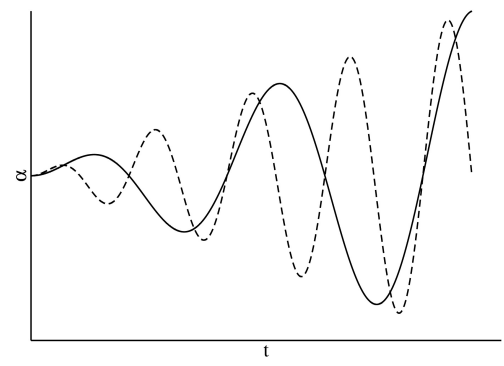

(a) $t-\alpha$



(b) $\alpha-\dot{\alpha}-\ddot{\alpha}$

Figure 4. Angle of attack as a function of time and regressor space coverage for the spiral maneuver



(a) $t-\alpha$



(b) $\alpha-\dot{\alpha}-\ddot{\alpha}$

Figure 5. Angle of attack as a function of time and regressor space coverage for the DC spiral maneuver

\section{Piecewise Linear Spirals (PWLS)}

A common limitation of both the chirps and spirals maneuvers is that they result in poor regressor space coverage of very low frequency motions. This is particularly true for static data which is essentially not covered except for the initial data point at $t=0$. One possibility is to extend the low frequency segment by reducing the frequency growth rate to obtain data for a very low frequency spiral maneuver. However, such an approach has two major shortcomings. The first is the assumption that static data can be predicted from information contained in a maneuver with very low frequencies, an assumption which does not necessarily need to be valid. The second is, that even if the static data can be predicted from very low frequency maneuvers, the time needed to execute such a maneuver is excessive.

A possible solution to this issue is a maneuver consisting of linear segments. As such, this maneuver will always have the second derivative $\ddot{\alpha}=0$ and will cover regions of $\alpha-\dot{\alpha}$ regressor space that cannot 
be covered with the standard chirp or spiral maneuver. The ideal shape of the PWLS maneuver is a step maneuver. Such a maneuver produces discontinuities in the input signal which captures the system's complete frequency content. ${ }^{25,26}$ This maneuver is used as a training maneuver for the Volterra type of $\mathrm{ROM}^{26,27}$ and has excellent predictive capabilities in linear aerodynamics applications. There are however technical and numerical difficulties associated with this type of signal, particularly when the amplitude is large.

The piecewise linear spiral, PWLS, maneuver proposed here is derived from the spiral maneuver. Figure 6 shows the regressor space coverage of two such maneuvers. Static data is located along the line $\dot{\alpha}=0$. The $\alpha=$ const segment of the PWLS maneuver specifically has $\dot{\alpha}=\ddot{\alpha}=0$, while aerodynamic loads are initially unsteady and are a function of time. Therefore the length of this segment must be sufficiently long to allow the system to reach steady state values.

The DC PWLS maneuver is essentially a PWLS maneuver with an initial offset and is based on DC spiral maneuver introduced previously. Two examples of the resulting regressor space coverage are shown in figure 7. Like the DC chirp and spiral maneuvers, the regressor space is not symmetric about $\alpha_{0}$, instead it extends from $\alpha=0$ to the maximum amplitude. The motivation for using this maneuver is that is gives controllability of the regressor space coverage in the $\ddot{\alpha}=0$ plane which contains the static data.

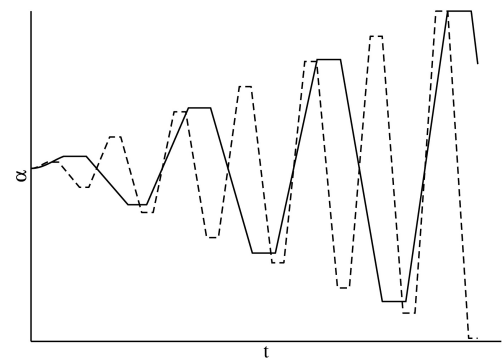

(a) $t-\alpha$



(b) $\alpha-\dot{\alpha}$

Figure 6. Angle of attack as a function of time and regressor space coverage for the PWLS maneuver

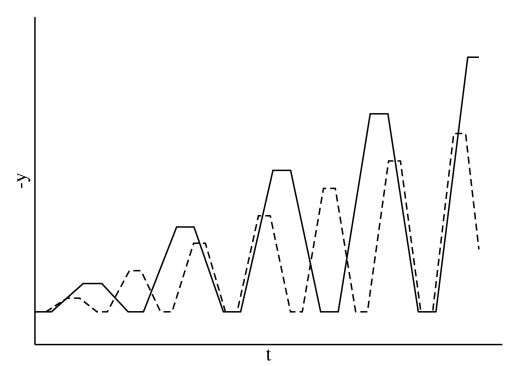

(a) $t-\alpha$

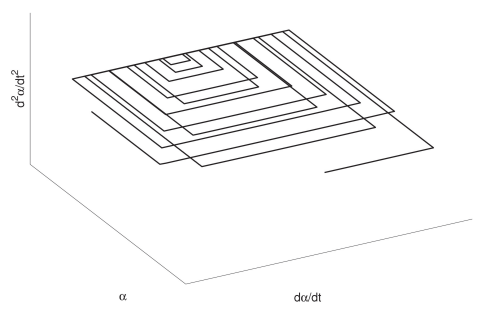

(b) $\alpha-\dot{\alpha}$

Figure 7. Angle of attack as a function of time and regressor space coverage for the DC PWLS maneuver

\section{Schroeder maneuver}

The Schroeder maneuver is a multi-sine frequency sweep consisting of multiple cosine terms with a specified phasing $^{28}$ that is optimized for a flat PDS spectrum over a specified frequency range. This wide band training signal was previously used by Murphy et al. ${ }^{23}$ and Goertz et al. ${ }^{14}$ for aircraft system identification with $\mathrm{S} \& \mathrm{C}$ applications. The angle of attack is specified as follows

$$
\alpha(t)=A \sum_{k=1}^{N} \sqrt{\frac{1}{2 N}} \cos \left(\frac{2 \pi k t}{T}-\frac{\pi k^{2}}{N}\right) .
$$


An example of the resulting angle of attack as a function of time and regressor space coverage are shown in figure 8 .

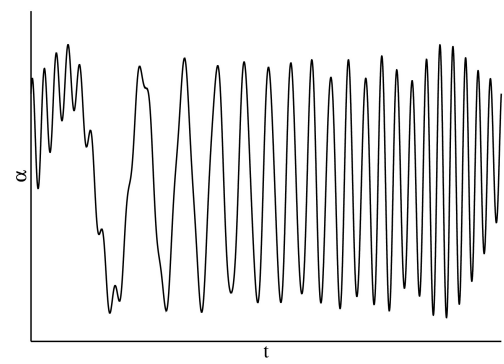

(a) $t-\alpha$

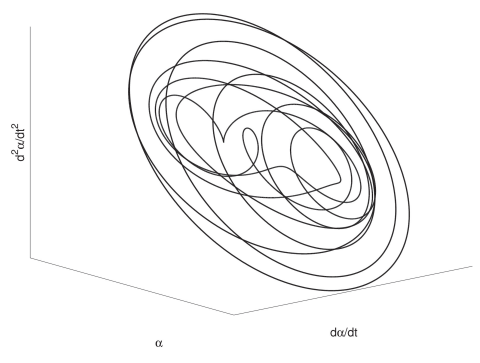

(b) $\alpha-\dot{\alpha}-\ddot{\alpha}$

Figure 8. Angle of attack as a function of time and regressor space coverage for the Schroeder maneuver

The Schroeder maneuver has three parameters that enable direct control of the regressor space coverage. These are maneuver amplitude, $A$, the maneuver length, $T$, and the number of frequencies in the maneuver, $N$. Changing the amplitude scales all three dimensions with the same scaling factor, which is identical to the previous maneuvers. Changing the maneuver length $T$ scales the rate and acceleration such that when $T$ is increased, rate and acceleration decrease. Figure 9 shows the effect of varying $T$ on the Schroeder maneuver in the three planes of the regressor space. Including higher frequencies in the Schroeder maneuver by increasing the value of $N$, also alters the shape of the angle of attack curve. In addition, since the value of the first and second derivative strongly depends on frequency, including higher frequencies extends the limits of rate and acceleration coverage by the maneuver. Figure 10 shows the effect of $N$ on the Schroeder maneuver in the three planes of the regressor space. By altering $T$ and $N$ simultaneously one can design the Schroeder maneuver covering the same regressor space while altering the density of the coverage within the space. However, for dense coverage the total maneuver time is significantly increased.

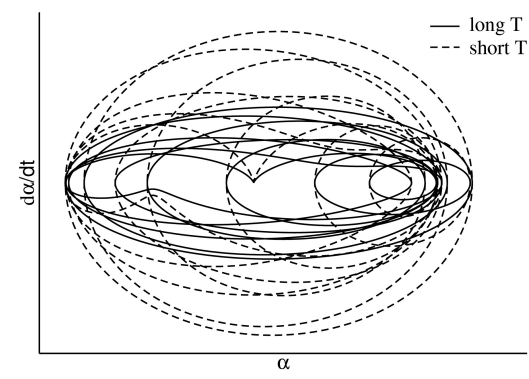

(a) $\alpha-\dot{\alpha}$



(b) $\alpha-\ddot{\alpha}$



(c) $\dot{\alpha}-\ddot{\alpha}$

Figure 9. Effect of varying the length $T$ on the resulting regressor space coverage for the Schroeder maneuver

A limitation of the Schroeder maneuver is that it uses most of its resources on the perimeter of the regressor space leaving the low rate and acceleration space poorly covered (see figures 9(a) and 10(a)). This can be, to a certain extent, reduced by lowering the value of $N$ and making the maneuver longer. Ultimately, for $N=1$ the Schroeder maneuver reduces to a simple sinusoidal wave and for $N=2$ the resulting maneuver is similar to the spiral maneuver introduced previously. A second limitation is that the Schroeder maneuver does not start from the $\alpha=0$ condition, posing difficulties on the start-up state.

\section{B. Translational motion}

The primary difference between rotational and translational motion is that for rotational motion the angle of attack, $\alpha$, is the primary variable, while for translation an effective angle of attack must be derived from the motion. In the case of a two-dimensional plunging airfoil with the $y$-axis defined normal to the chord, it 


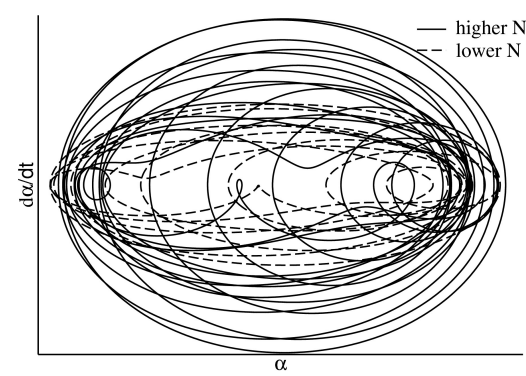

(a) $\alpha-\dot{\alpha}$



(b) $\alpha-\ddot{\alpha}$



(c) $\dot{\alpha}-\ddot{\alpha}$

Figure 10. Effect of varying $N$ on the resulting regressor space coverage for the Schroeder maneuver

is the first derivative of the $y$-axis motion that determines the effective angle of attack according to

$$
\alpha_{e f f}=\arctan \frac{-\dot{y}}{v_{\infty}}
$$

Therefore, the maneuvers defined in previous section can be used to either directly prescribe a translational motion in the $y$-axis direction, or to prescribe the first derivative, $\dot{y}$. The advantage of the first approach is that all of the motions can be determined analytically, while the disadvantage is the indirect control of $\alpha_{e f f}$. The advantage of the second approach is direct control of $\alpha_{e f f}$, while the drawback is that some motions are determined by numerical integration. Other disadvantages of the second approach include: the motion depends on the freestream velocity, $v_{\infty}$ which can make the integration difficult if $v_{\infty}$ is time dependent, and some of the integrated motions result in relatively large displacements making them unsuitable for wind tunnels. The following sections describe the various maneuvers using the integrated motions approach. In all cases, the freestream velocity, $v_{\infty}$, is assumed constant.

\section{Integrated Chirp}

The primary issue with implementing a chirp motion to prescribe a translation is that the amplitude of the first derivative, which determines the value of $\alpha_{\text {eff }}$, is not constant, but grows with the frequency of the motion. This results in very low $\alpha_{e f f}$ at low frequencies making it difficult to examine the nonlinear aerodynamics in this region. This can be avoided by using the chirp motion to explicitly prescribe the first derivative of the translational motion and consequently $\alpha_{e f f}$. The translational motion in the $y$-axis direction is then defined as

$$
\begin{aligned}
& y(t)=A \int \sin \left(\omega t^{2}\right) \\
& \dot{y}(t)=A \sin \left(\omega t^{2}\right) .
\end{aligned}
$$

This integral, known as the Fresnel integral, does not have an analytical form and has to be solved numerically. The time history of displacement is then a function of the amplitude of $\alpha_{e f f}$ and the freestream velocity, $v_{\infty}$. Figure 11 shows examples of two Fresnel integrals resulting in an $\alpha_{\text {eff }}$ with a chip motion shape. A disadvantage of the Fresnel chirp is that the initial displacement can reach values that are unobtainable by wind tunnel experiments, however this is less of an issue for CFD simulation.

The Fresnel integral must be transformed into the primitive variables $\alpha_{e f f}, \dot{\alpha}_{e f f}, \ddot{\alpha}_{e f f}$. Since the primary motivation for the Fresnel chirp is direct control over the shape and values of $\alpha_{\text {eff }}$, it would be natural to investigate the Fresnel chirp regressor space defined in terms of $\alpha_{e f f}=\arctan \left(-\dot{y} / v_{\infty}\right)$ and its first and second derivatives. This regressor space will retain the shape of the original motion used for the integration, in this case of the chirp motion shown in figure 2.

As in the case of the integrated chirp maneuver, the integrated DC chirp motion does not have an analytical expression and must be computed numerically. The equations describing the translational motion 


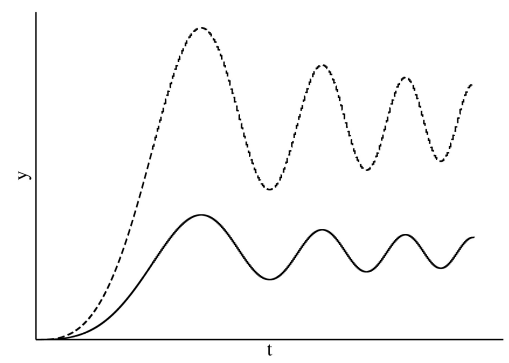

(a) $t-y$



(b) $y-\dot{y}-\ddot{y}$

Figure 11. Displacement as a function of time and regressor space coverage for the Fresnel chirp

in the $y$-axis direction are

$$
\begin{aligned}
& y(t)=\frac{A}{2} \int\left(1-\cos \left(\omega t^{2}\right)\right) \\
& \dot{y}(t)=\frac{A}{2}\left(1-\cos \left(\omega t^{2}\right)\right) .
\end{aligned}
$$

Figure 12 shows two displacements that result in an $\alpha_{\text {eff }}$ with DC chirp shapes. Due to the constant term in equation 9 , the resulting motion is a superposition of an oscillatory motion and a linear increase. Similarly to the integrated chirp, this motion has limited applications in wind tunnels. The integrated DC chirp must also be transformed to the primitive variables $\alpha_{e f f}, \dot{\alpha}_{e f f}, \ddot{\alpha}_{e f f}$. Doing so will result in a regressor space coverage that retains the shape of the DC chirp motion shown in figure 3.

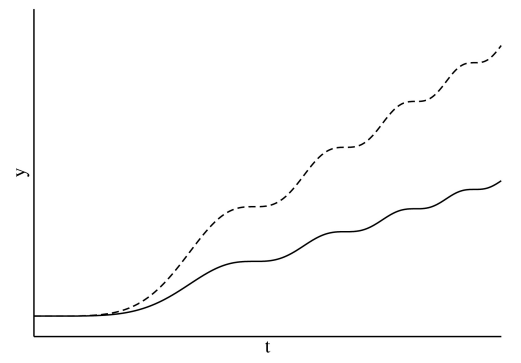

(a) $t-y$

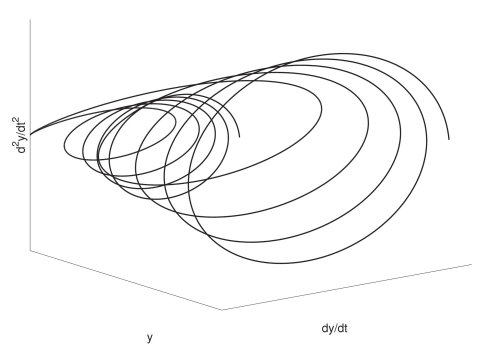

(b) $y-\dot{y}-\ddot{y}$

Figure 12. Displacement as a function of time and regressor space coverage for the integrated DC chirp

\section{Integrated Spiral Maneuver}

For an integrated spiral maneuver the translational motion in the $y$-axis direction is defined as an integral of the original spiral motion (Eq. 3) according to

$$
y(t)=\int A t \sin (\omega t)=A\left(\frac{1}{\omega^{2}} \sin (\omega t)-\frac{t}{\omega} \cos (\omega t)\right) .
$$

Two examples of the $y$-axis displacement resulting in an $\alpha_{\text {eff }}$ with a spiral shape are shown in figure 13(a).

Like the previous integrated maneuvers, the integrated spiral must be transformed to the primitive variables $\alpha_{e f f}, \dot{\alpha}_{e f f}, \ddot{\alpha}_{e f f}$. The regressor space will then retain the shape of the spiral motion shown in figure 4. For this maneuver the shape of the regressor space is the same as the original displacement in $y$, and therefore there is not any advantage of using the integrated spiral motion unless one is trying to keep the 
induced angle of attack consistent with the original spiral motion. It will, however, have a different shape if $v_{\infty}$ is dependent on time; in this case having a simple term in the integration may be advantageous.

The integrated DC spiral maneuver is defined in a similar fashion as above. The translation motion in the $y$-axis direction is then defined as

$$
\alpha(t)=\int \frac{A}{2} t(1-\cos (\omega t))=\frac{A}{2}\left(\frac{t^{2}}{2}-\frac{t}{\omega} \sin (\omega t)-\frac{1}{\omega^{2}} \cos (\omega t)+\frac{1}{\omega^{2}}\right) .
$$

Two examples of the $y$-axis displacement resulting in an $\alpha_{e f f}$ with a DC spiral shape are shown in figure 14. Due to the linear term in equation 12, the motion consists of a superposition of an oscillatory motion and a quadratic increase, making it unsuitable for wind tunnels. Like the previous integrated maneuvers, the integrated DC spiral must be transformed to the primitive variables $\alpha_{e f f}, \dot{\alpha}_{e f f}, \ddot{\alpha}_{e f f}$ and the resulting motion retains the shape of the DC spiral motion shown in figure 5.



(a) $t-y$

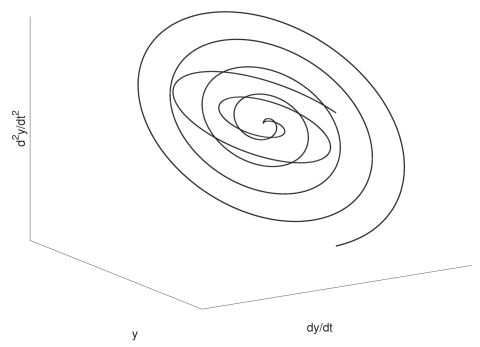

(b) $y-\dot{y}-\ddot{y}$

Figure 13. Displacement as a function of time and regressor space coverage for the integrated spiral motion

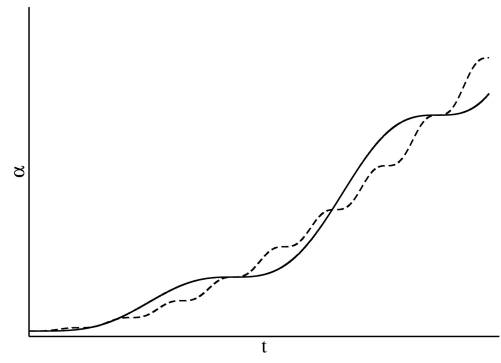

(a) $t-y$

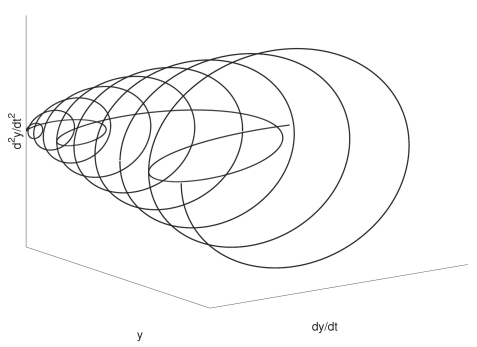

(b) $y-\dot{y}-\ddot{y}$

Figure 14. Displacement as a function of time and regressor space coverage for the integrated DC spiral

\section{Integrated PWLS Maneuver}

The integrated PWLS maneuver consists of a sequence of linear and quadratic segments. Two examples of the integrated PWLS maneuver and the regressor space coverage are shown in figure 15. The advantage of the integrated PWLS over the PWLS maneuver for translational motion is that the integrated PWLS induces a continuous $\alpha_{\text {eff }}$ while the PWLS induces step-wise functions of $\alpha_{e f f}$. Like the previous integrated maneuvers, the integrated PWLS must be transformed to the primitive variables $\alpha_{e f f}, \dot{\alpha}_{e f f}, \ddot{\alpha}_{e f f}$ and the resulting motion retains the shape of the original PWLS motion shown in figure 6.

The integrated DC PWLS is a superposition of the integrated PWLS maneuver and a linear increase in time. Figure 16 shows the displacement in the $y$-axis direction as a function of time and the resulting regressor space coverage for two integrated DC PWLS maneuvers. Due to large displacement values caused by a constant term in the integration, this motion has limited use in wind tunnels. Transforming the 
integrated DC PWLS maneuver into the primitive variables $\alpha_{e f f}, \dot{\alpha}_{e f f}$, and $\ddot{\alpha}_{e f f}$ retains the shape of the original DC PWLS maneuver shown in figure 7

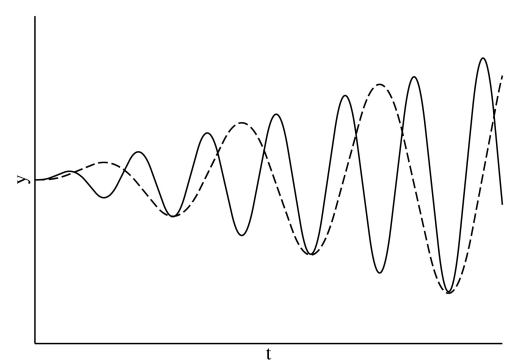

(a) $t-y$

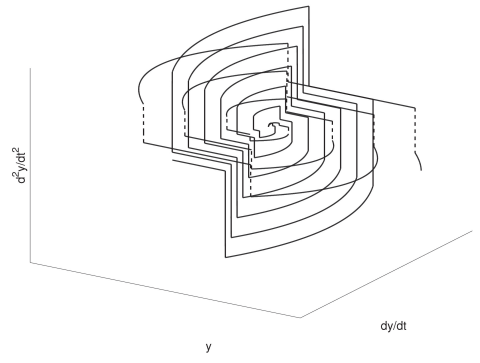

(b) $y-\dot{y}-\ddot{y}$

Figure 15. Displacement as a function of time and regressor space coverage for the integrated PWLS maneuver

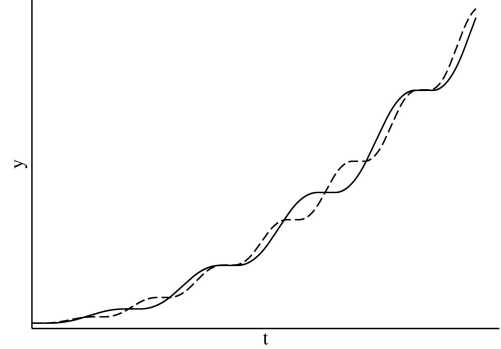

(a) $t-y$

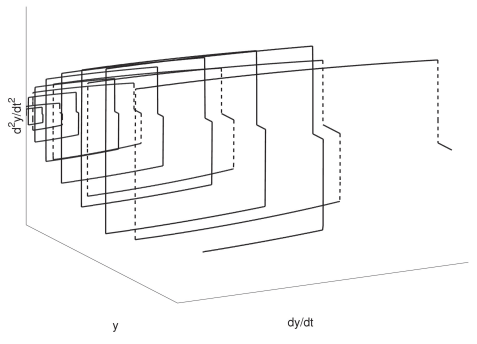

(b) $y-\dot{y}-\ddot{y}$

Figure 16. Displacement as a function of time and regressor space coverage for the integrated DC PWLS maneuver

\section{Integrated Schroeder Maneuver}

The $y$-axis displacement of integrated Schroeder maneuver is defined as

$$
y(t)=\int \sum_{k=1}^{N} \sqrt{\frac{1}{2 N}} \cos \left(\frac{2 \pi k t}{T}-\frac{\pi k^{2}}{N}\right)=\sum_{k=1}^{N} \sqrt{\frac{1}{2 N}} \frac{T}{2 \pi k} \sin \left(\frac{2 \pi k t}{T}-\frac{\pi k^{2}}{N}\right) .
$$

An example of the $y$-axis displacement and the resulting regressor space coverage is shown in figure 17 . The integrated Schroeder maneuver provides random coverage of the regressor space, however as in the case of the Schroeder maneuver, most of the coverage is concentrated on the perimeter of the regressor space.

The integrated Schroeder maneuver transformed into $\alpha_{e f f}$ variables retains the shape of the original Schroeder maneuver shown in figure 6. The main advantage of using the regressor space defined in terms of $\alpha_{\text {eff }}$ rather than $y$-axis displacement is that the shape is more compact and the regressor space coverage is more regular, which can influence the quality of the ROM interpolation.

\section{Computational Methodology}

\section{A. Flow Solver}

Computations were performed using the commercially available flow solver Cobalt, ${ }^{29}$ which solves the unsteady, three-dimensional, compressible Navier-Stokes equations. Cobalt is a cell-centered, finite volume 


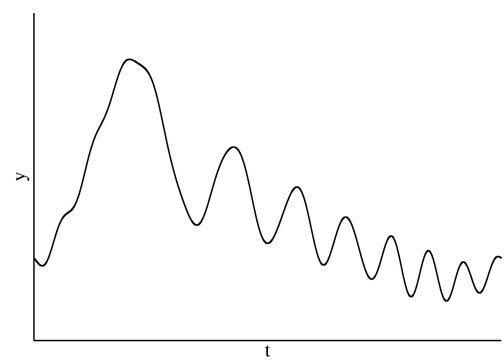

(a) $t-y$

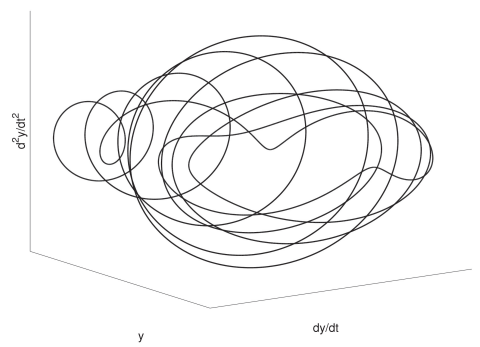

(b) $y-\dot{y}-\ddot{y}$

Figure 17. Displacement as a function of time and regressor space coverage for the integrated Schroeder maneuver

based code applicable to arbitrary cell topologies including prisms, tetrahedra and hexahedrals. Secondorder accuracy in space is achieved using the exact Riemann solver of Gottleib and Groth, ${ }^{30}$ and gradient reconstruction using a least squares technique. To advance the discretized system a point-implicit method using analytic first-order inviscid and viscous Jacobians is used. A Newton sub-iteration method is used in the solution of the system of equations to improve time accuracy of the point-implicit method. The method is second-order accurate in time. Tomaro et al. ${ }^{31}$ converted the code from explicit to implicit, enabling CFL numbers as high as $10^{6}$. Cobalt is cast in an arbitrary Lagrangian-Eulerian formulation, and therefore, has the ability to compute rigid-body motion. ${ }^{32}$ Cobalt has both free and specified 6-degree-of-freedom rigid-body motion capabilities. Rigid-body motion reorients the grid without deforming it using a transformation matrix and a translation vector describes the motion of the grid. Due to the two-dimensional nature of the simulations Unsteady Reynolds Averages Navier-Stokes (URANS) was chosen for turbulence closure over Detached Eddy Simulation (DES), which was primarily developed for three-dimensional turbulence. Time-dependent static and dynamic computations were performed using the Shear Stress Transport (SST) technique proposed by Menter. ${ }^{33}$ The SST model is known to give reliable predictions of two-dimensional flow separation due to adverse pressure gradients. The SST model is a blend between the standard $k-\epsilon$ and $k-\omega$ models, where the former is implemented in the outer region and the latter in the near wall region. For each region standard transport equations are solved for either $k$ and $\epsilon$ or $k$ and $\omega$.

\section{B. Computational Setup}

Simulations were conducted using the standard NACA0012 profile. The computational domain was rectangular in shape with the airfoil geometry centrally located. The minimal distance from the body to each of the outer boundaries was $20 c$. The no-slip adiabatic wall boundary condition was employed at the body surface and the modified Riemann-invariant condition was implemented at the farfield boundary. All simulations were performed at $R e_{c}=9.0 \times 10^{6}, M_{\infty}=0.20$ for direct comparison to experimental data presented in Abbot and Von Doenhoff. ${ }^{34}$ For static simulations the computational domain was initialized to free stream conditions and for dynamic simulations the computational domain was initialized using the appropriate static solution. To ensure that the solution was converged at every time step, five Newton sub-iterations were used for all dynamic simulations.

\section{Mesh Refinement and Time Step Study}

The mesh used for the current study was created using Solidmesh 2D, ${ }^{35}$ which is an Advancing Front Local Reconnection (AFLR) unstructured grid generator. A total of three meshes with total cell counts of 41,758, 83,018, and 176,948, respectively, were created for the current study. Schematics of the medium density mesh showing the unstructured outer region and the prismatic boundary layer are shown in figure 18 The first node height was chosen such that the average $y^{+}$was below 1.0, which ensured an adequate volume mesh that correctly models the boundary layer. To ensure that both spatial and temporal independence have been achieved, static simulations were performed on all three meshes at a constant angle of attack of 




(a) Unstructured mesh in outer flow region

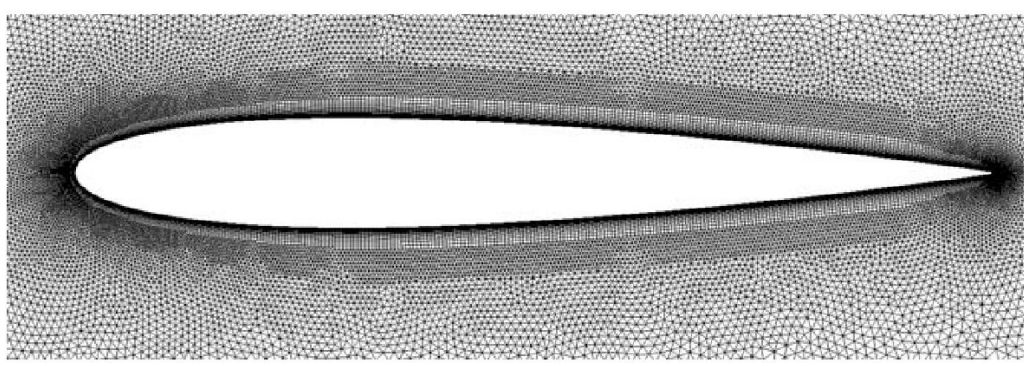

(b) prismatic boundary layer region

Figure 18. Schematics of the medium density mesh

$\alpha=15 \mathrm{deg}$ for dimensionless time steps ranging from $\Delta t^{*}=0.08-0.01$, where $\Delta t^{*}$ is defined by,

$$
\Delta t^{*}=\frac{\Delta t v_{\infty}}{c}
$$

Comparisons of the predicted lift and drag coefficients showed that the 83,018 cell medium density mesh in conjunction with $\Delta t^{*}=0.01$ was sufficient for the static simulations. It was then assumed that these parameters combined with 5 sub-Newton iterations were also sufficient for the dynamic simulations.

\section{S\&C Aerodynamic Loads Modeling - Radial Basis Function, RBF}

The goal of the CFD calculations is to produce training data that can be used to build a reliable ROM. The ROM implemented for the current study is based on Radial Basis Functions, RBF. Previous research has primarily implemented the constant parameter interpolate designed specifically for aircraft system identification known as SIDPAC. ${ }^{12}$ SIDPAC is a least square regression based method that generates an explicit relationship between the aerodynamic force and state variables. This code has been extensively used at USAFA however, Jeans ${ }^{13}$ in his extensive study of an aircraft geometry showed that this method is incapable of generating an accurate low-order model of the aerodynamic loads based on the analysis of a single training maneuver. For the current application RBF appeared to be a better option. Based on these conclusions as well as on the preliminary tests with the NACA0012 airfoil and SIDPAC, which showed limited reliability, it was decided to use Radial Basis Functions for ROM generation.

Radial Basis Functions are a popular choice as a non-parametric interpolant used, for example, in aerodynamic shape optimization and mesh deformation. ${ }^{36-38}$ The classical RBF interpolation is a linear combination of basis functions radial around point $x$

$$
y(x)=\sum_{l=1}^{N} \gamma_{l} \phi\left(c\left|x-x_{l}\right|\right)
$$

were $\phi$ is a basis function and the term $\left(\left|x-x_{l}\right|\right)$ is the Euclidian distance and $c$ is a shape factor. The coefficients $\gamma$ are found from the condition that the RBF representation interpolates exactly at the control points, which in this application are the aerodynamic values of the training maneuvers. Some of the most common basis functions, their effect on predictive accuracy and their comparison to other metamodeling models can be found, for example, in Krishnamurthy. ${ }^{39}$ The basis function used during this study is a multiquadratic function developed by Hardy. ${ }^{40}$

\section{Results and Discussion}

All of the dynamic CFD simulations were performed at the Arctic Region Supercomputing Center (ARSC) on Midnight, a Sun cluster comprised of 2312 Opteron processors with a 68 TB Lustre file system. The calculations included the static and dynamic validation data as well as simulations of each of the training maneuvers described in Section II. 


\section{A. Validation Data}

\section{Static calculations}

Static simulations were completed at angles of attack ranging from $\alpha=0$ deg to $\alpha=20 \mathrm{deg}$ in 2 deg increments. Lift and pitching moment coefficient predictions are shown in figure 19. Overall, the lift coefficients predicted by the SST model compare favorably to published data in Abbot and Von Doenhoff, ${ }^{34}$ however, a key difference is that the SST model delayed stall beyond $\alpha=16$ deg by $1-2$ degrees. As shown in figure 19(a), once the wing did stall the SST model correctly predicted the corresponding drop in lift coefficient due to massive flow separation near the leading edge of the upper surface.

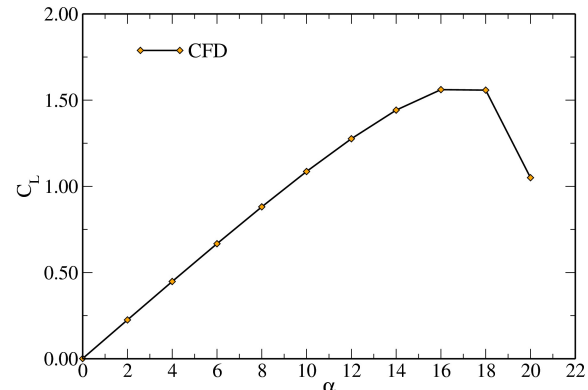

(a) Time average lift coefficient

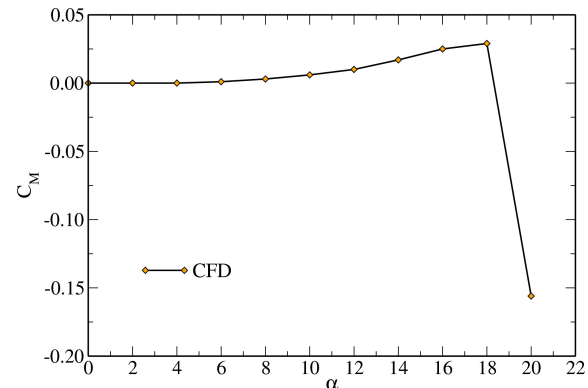

(b) Time average pitching moment coefficient

Figure 19. Static SST predictions of lift and pitching moment coefficient for the NACA0012 airfoil at $R e_{c}=$ $9 \times 10^{6}, M_{\infty}=0.2$

\section{Dynamic Calculations}

The dynamic pitching validation data consists of sinusoidal motions about $\alpha_{0}=10 \mathrm{deg}$ at frequencies of $f=0.5 \mathrm{~Hz}, 1 \mathrm{~Hz}$ and $2 \mathrm{~Hz}$ and amplitudes of $A=5 \mathrm{deg}$ and 10deg. As shown in figure 20, the 5deg amplitude motion results in linear aerodynamic behavior, while the 10deg amplitude motion results in nonlinear aerodynamics at the lower frequencies when the angle of attack is greater than $\alpha=18 \mathrm{deg}$. In addition, the validation data shown in figure 20(b) varies from strongly nonlinear to less nonlinear as the frequency of the oscillatory motion is increased. A single reduced order model that can predict the linear and nonlinear aerodynamic behavior for a variety of frequencies and amplitudes such as these must be very robust.

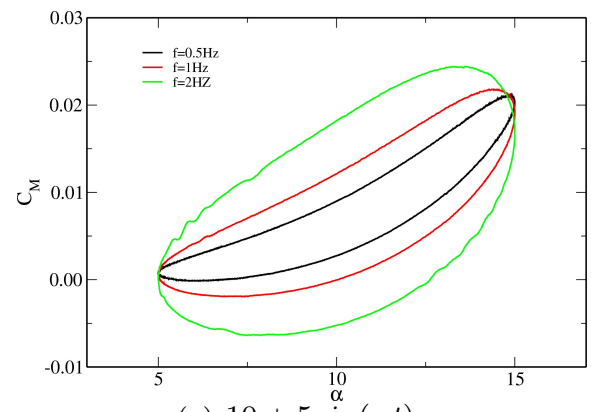

(a) $10+5 \sin (\omega t)$

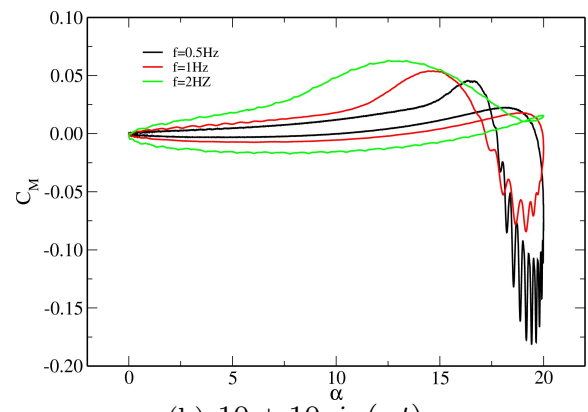

(b) $10+10 \sin (\omega t)$

Figure 20. SST predictions of pitching moment for several dynamic pitching simulation of the NACA0012 airfoil at $R e_{c}=9 \times 10^{6}, M_{\infty}=0.2$

The dynamic plunging validation data consists of a simple oscillatory motion and a more complicated Fresnel chirp maneuver. The oscillatory motion had an amplitude of $\alpha_{e f f}=10 \mathrm{deg}$ and frequency of $f=$ $0.85 \mathrm{~Hz}$. CFD predictions of pitching moment for each maneuver are shown in figure 21. Both maneuvers result in linear and nonlinear aerodynamics, and are therefore excellent tests of the ability of a single ROM. 


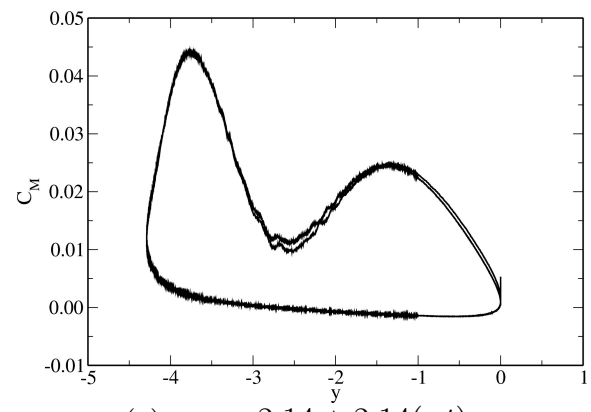

(a) $y=-2.14+2.14(\omega t)$



(b) Fresnel chirp

Figure 21. SST predictions of dynamic plunging motions, pitching moment, NACA0012 airfoil at $R e_{c}=9 \times 10^{6}$, $M_{\infty}=0.2$

\section{B. Training Maneuver Simulations}

\section{Pitching Motion}

The various training maneuvers described in Section II were used to specify pitching motion of the NACA0012 airfoil about $x=0.25 \mathrm{c}$. For all of the simulations, the initial angle of attack was constant at $\alpha_{0}=10 \mathrm{deg}$. The goal is to evaluate which training maneuver is best at producing training data for the ROM. The resulting data is also examined for the presence of nonlinear aerodynamics. For each specific training maneuver simulations are completed at various amplitudes and frequencies such that the desired regressor space is sufficiently covered.

Chirp Maneuvers Five chip maneuvers were completed with frequency increasing linearly from $f=0 \mathrm{~Hz}$ to $6 \mathrm{~Hz}$ at amplitudes of $A=2,4.5,7.5$ and 10deg. The resulting lift and pitching moment coefficients as a function of $\alpha$ are shown in figure 22. As evident in the figure, most of the resulting aerodynamic data is linear in $\alpha$ even at 20deg attack. This is because for most of the maneuvers the frequency is sufficiently large that the airfoil behaves linearly even at extreme angles of attack. However, for the $A=10 \mathrm{deg}$ maneuver the lift and pitching moment coefficients do behave nonlinearly when the frequency is approximately less than $f=0.5 H z$.

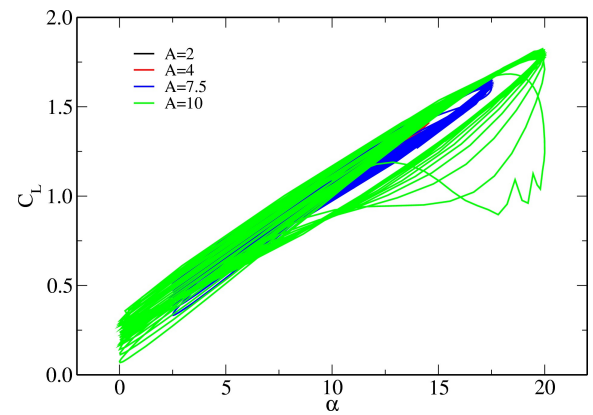

(a) $t-C_{L}$

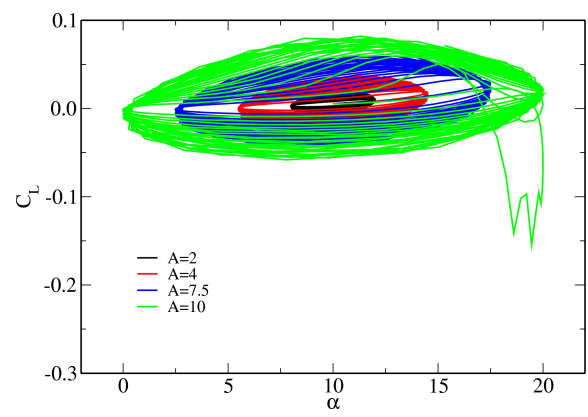

(b) $t-C_{M}$

Figure 22. SST predictions of lift and pitching moment for chirp motions of a pitching NACA0012 airfoil

Spiral Maneuvers Five spiral motions with a maximum angle of attack of $\alpha=22 d e g$ were completed at $f=0.1,0.5,1,2$ and $3 \mathrm{~Hz}$. The resulting lift and pitching moment predictions are shown in figure 23. Compared to figure 22 the spiral motions produced considerably more nonlinear data then the chirp motion. This is primarily due to the fact that the frequency is constant for each individual spiral maneuver and therefore is it possible to explicitly specify extreme angles of attack at low frequencies.

A PWLS maneuver based on a $2 \mathrm{~Hz}$ spiral maneuver was also completed. As previously noted, the main advantage of this maneuver is that it is located in the $\ddot{\alpha}=0$ plane, and therefore contains static data. The resulting lift and pitching moment coefficient predictions are also shown in figure 23. Similarly to the low 
frequency spiral maneuvers, the PWLS maneuver captures a lot of nonlinearity in both lift and pitching moment.

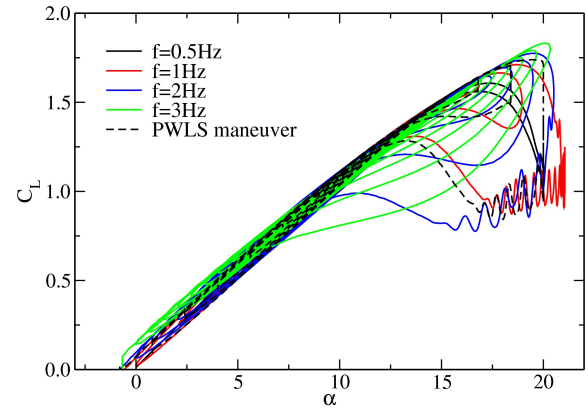

(a) $t-C_{L}$

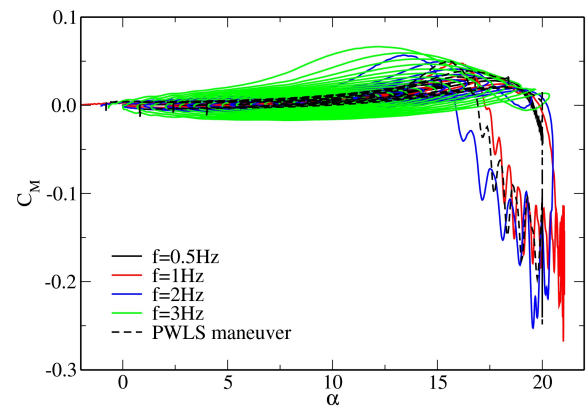

(b) $t-C_{M}$

Figure 23. SST predictions of lift and pitching moment for spiral motions of a pitching NACA0012 airfoil

Schroeder Maneuvers Three Schroeder maneuvers were completed with the following combinations of maneuver length, $T$, and number of frequencies, $N$ : (a) $N=15, T=3 \mathrm{sec}$, (b) $N=15, T=10 \mathrm{sec}$ and (c) $N=30$ and $T=5 \mathrm{sec}$. Resulting CFD predictions of lift and pitching moment coefficient for each of these maneuvers is shown in figure 24. The maneuvers were designed to provide broad coverage of the regressor space at both low and high frequencies. However, the nonlinear aerodynamic content is similar to that of the chirp maneuvers shown in figure 22.

The main conclusion from the above comparisons is that of the maneuver investigated, spiral maneuvers contain the most low frequency nonlinear aerodynamic behavior (primarily because it is specified explicitly), but are also computationally most demanding.

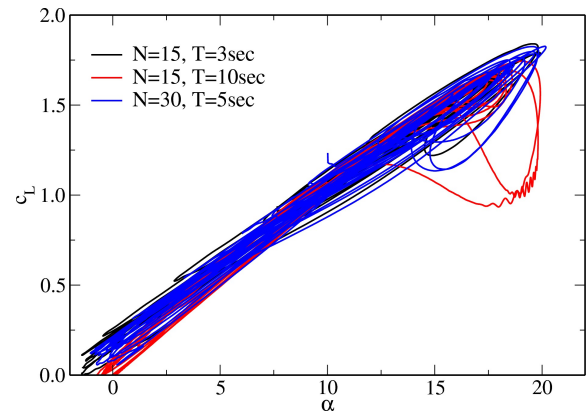

(a) $t-C_{L}$



(b) $t-C_{M}$

Figure 24. SST predictions of lift and pitching moment for Schroeder motions of a pitching NACA0012 airfoil

\section{Plunging}

The various training maneuvers described in Section II were also used to specify plunging motion of the NACA0012 airfoil. The plunging motion was achieved by displacing the entire mesh in the $y$-axis direction. For all of the simulations the initial displacement was zero and the airfoil was fixed at an initial angle of attack, $\alpha_{0}=0 \mathrm{deg}$. The freestream velocity vector was also held constant. The training maneuvers tested for plunging motion included: the chirp motion and Fresnel chirp motion, integrated spiral motion, PWLS and integrated PWLS maneuver.

Chirp Motion Four chirp maneuvers were completed with frequency increasing linearly from $f=0 \mathrm{~Hz}$ to $6 \mathrm{~Hz}$ at displacement amplitudes of $A=5,14,23$ and $32 \%$ of the airfoil cord. Predicted lift and pitching moment coefficients are shown in figure 25. Lift and pitching moment behaved linearly for all of the prescribed chirp motions. This is because at low frequencies the values of $\alpha_{\text {eff }}$ are too small for the airfoil to behave 
nonlinearly, and at higher frequencies that induce values of $\alpha_{\text {eff }} 18 \mathrm{deg}$ the frequency is sufficiently high that any nonlinear behavior is eliminated.

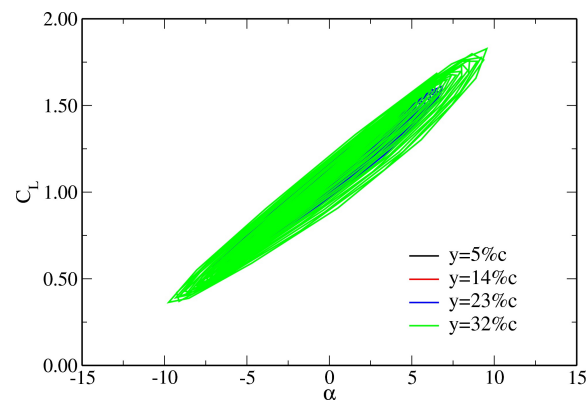

(a) $C_{L}$



(b) $C_{M}$

Figure 25. SST predictions of lift and pitching moment for chirp motions of a plunging NACA0012 airfoil

Fresnel Chirp Motion Several Fresnel chirps were designed such that the maximum induced angle of attack ranged from $\alpha_{e f f}=1,4,7$ and 10deg. Predicted lift and pitching moment coefficients are shown in figure 26. In contrast to the chirp maneuvers, the Fresnel chirps excited the airfoil's nonlinear behavior at low frequencies. However, the Fresnel chirp also inherited the chirp's poor qualities at very low frequencies. The data in figure 26 is similar to the chirp pitching motion predictions in figure 22, with most of the maneuver being in the linear region of lift and pitching moment.



(a) $C_{L}$

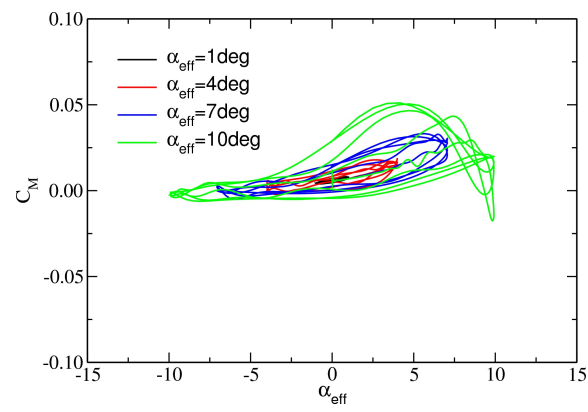

(b) $C_{M}$

Figure 26. SST predictions of lift and pitching moment for Fresnel chirp motions of a plunging NACA0012 airfoil

Integrated Spiral Motion Three integrated spiral motions with a maximum induced angle of attack of $\alpha_{\text {eff }}=10 \mathrm{deg}$ were completed at specified frequencies of $f=0.5,1$ and $2 \mathrm{~Hz}$. Predicted lift and pitching moment coefficients are shown in figure 26. The resulting lift and moment predictions are similar to those of the Fresnel chirp motion results because the lowest frequency of the integrated spiral maneuver was $f=0.5 \mathrm{~Hz}$, which was similar to the lowest frequency of the Fresnel chirp that resulted in sufficient angles of attack for nonlinear aerodynamic behavior.

InTEGRATED PWLS Motion The last training maneuver computed for plunging was the integrated PWLS maneuver. For comparison, a standard PWLS plunging maneuver was also completed. Plotted in figure 28 are $y$-axis displacement and induced angle of attack for each maneuver. Defining the displacement using a PWLS motion resulted in an $\alpha_{e f f}$ distribution with step-wise input, while the integrated PWLS displacement resulted in a piece-wise linear distribution of $\alpha_{\text {eff }}$.

Predicted lift and pitching moment coefficients for each maneuver are shown in figure 29. For clarity, the data is plotted verses displacement rather then $\alpha_{e f f}$. Both maneuvers resulted in similar predictions of both lift and pitching moment as well as the time necessary for flow separation, suggesting that both maneuvers are equally suited for producing nonlinear low frequency data. Both training maneuvers also resulted in 


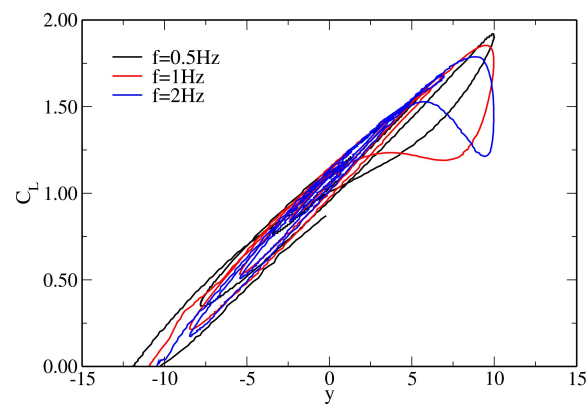

(a) $C_{L}$



(b) $C_{M}$

Figure 27. SST predictions of lift and pitching moment for integrated spiral motions of a plunging NACA0012 airfoil

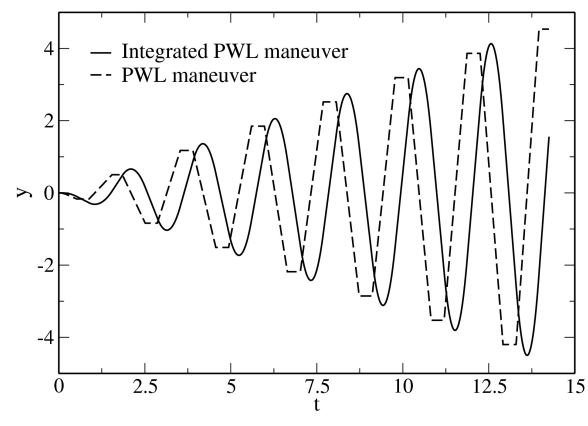

(a) $y-t$



(b) $\alpha_{e f f}-t$

Figure 28. A comparison of displacement and resulting $\alpha_{e f f}$ for the Integrated PWLS and PWLS maneuvers 
nonlinear aerodynamic behavior once the induced angle of attack was greater than $\alpha_{\text {eff }} \approx 18 \mathrm{deg}$. However, a key difference occurs in the transition between segments of constant $\alpha_{\text {eff }}$. The integrated PWLS transition is smooth in displacement and consequently the lift and pitch curves are smooth also. This transition was not smooth for the PWLS maneuver and resulted in sever discontinuities for both lift and pitching moment, making the data difficult to filter through a low/high pass filter.

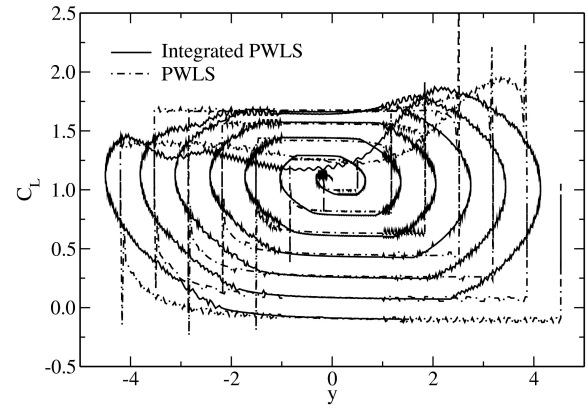

(a) $C_{L}$

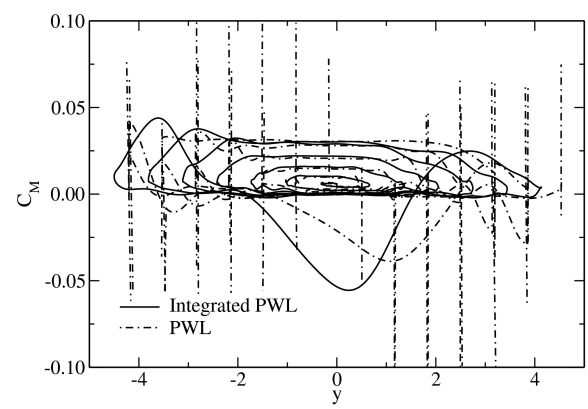

(b) $C_{M}$

Figure 29. SST predictions of lift and pitching moment for Integrated PWLS and PWLS motions of a plunging NACA0012 airfoil

The results from the plunging simulations confirmed that training maneuvers suitable for pitching motion may produce results without nonlinear content for plunging motion, as was the case for the chirp motion. Integrated motions can extend the nonlinear content in the training data, as was the case of the Fresnel chirp motion, or can produce training data that is easier to filter, as was the case of integrated PWLS maneuver.

\section{Lower-Order Aerodynamic Loads Modeling}

\section{Pitching Motion}

The aerodynamic loads predicted by the chirp, spiral, and Schroeder pitching maneuvers were each used as input to generate aerodynamic loads ROMs using radial basis functions. The resulting ROM was then used to predict both the static and dynamic validation data. The CFD training data were filtered through the low/high pass MLS filter ${ }^{41}$ to avoid modeling high-frequency oscillations. Some of the RBF ROM results also showed high-frequency oscillations, usually in regions of the regressor space with high gradients of lift or pitching moment, and were therefore filtered through the same low/high pass filter.

To obtain the maximum possible coverage of the regressor space the various training maneuvers within each maneuver class were combined into one larger training dataset prior to generating the ROM. For example, the five chirp training maneuver results were combined into one larger chirp training dataset. The same approach was also implemented for the spiral and Schroeder training maneuvers.

In the case of the spiral maneuvers the regressor space coverage was obtained by combining the spiral maneuvers at $f=0.5 \mathrm{~Hz}, 1 \mathrm{~Hz}, 2 \mathrm{~Hz}$ and $3 \mathrm{~Hz}$. The coverage was then completed by adding either the very low frequency $f=0.1 \mathrm{~Hz}$ spiral, or the PWLS maneuver. ROMs generated using either maneuver primarily affected the predicted static data. Figure 30 compares static predictions for an ROM dataset that includes either the $f=0.1 \mathrm{~Hz}$ spiral or the PWLS maneuver. Noticeable differences occur in the pitching moment predictions in figure 30(b), where the ROM that included the PWLS maneuver resulted in much better predictions. As a result, the final spiral ROM was generated using data from spiral maneuvers at $f=0.5 \mathrm{~Hz}, 1 \mathrm{~Hz}, 2 \mathrm{~Hz}$ and $3 \mathrm{~Hz}$ combined with the PWLS maneuver.

The resulting ROMs that were generated using training data from the various types of maneuvers were then used to predict the static and dynamic validation data presented in Section 1. Shown in figures 31, 32, 33 and 34 are comparisons of predicted lift and pitching moment by the the Spiral, Chirp and Schroeder based ROM to the dynamic CFD validation data. The dynamic validation data consists of sinusoidal motion about $\alpha_{0}=10 \mathrm{deg}$ at frequencies of $f=0.5 \mathrm{~Hz}, 1 \mathrm{~Hz}$ and $2 \mathrm{~Hz}$ and amplitudes of $A=5 \mathrm{deg}$ and 10deg. The variations in amplitude and frequency were such that it contains regions with both linear and nonlinear aerodynamic behavior making it a severe test case for the various ROMs. Spirals and chirp based ROMs generally resulted in good predictions of the aerodynamic loads for both lift and pitching moment. The Schroeder based ROM resulted in good predictions at lower frequencies and rather poor predictions at higher frequencies. This was 


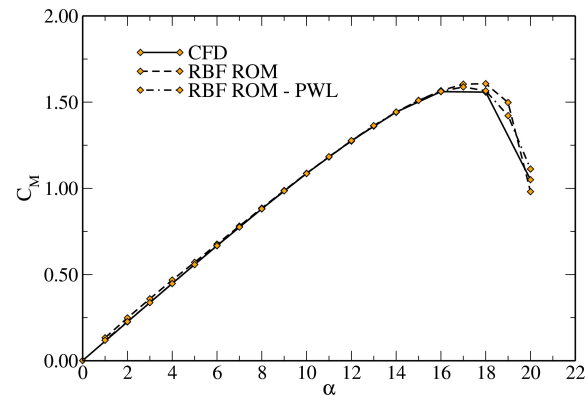

(a) $c_{L}$

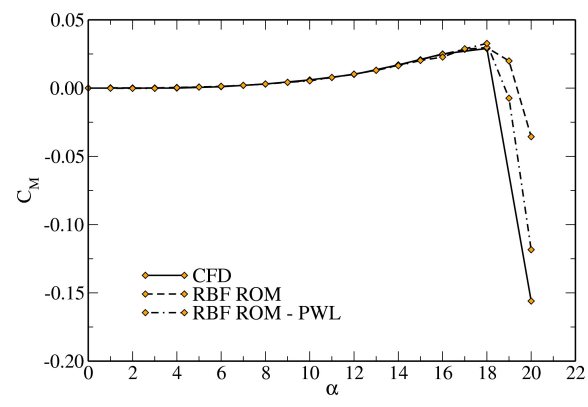

(b) $c_{M}$

Figure 30. A comparison of SST static lift and pitching moment predictions to RBF ROM predictions based on the spiral training maneuver dataset.

particularly true at a frequency of $f=2 \mathrm{~Hz}$. It is also important to note in figure $34(\mathrm{c})$ an oscillation in the spiral based pitching moment predictions at $2 \mathrm{~Hz}$. It is expected that the cause of this oscillation is the spiral maneuvers irregular coverage of the regressor space.



(a) $f=0.5 \mathrm{~Hz}$



(b) $f=1 H z$



(c) $f=2 H z$

Figure 31. A comparison of SST and ROM predictions of lift coefficient for a dynamic pitching maneuver of $\alpha=10 \pm 10 \sin (\omega t)$ at frequencies of $f=0.5 \mathrm{~Hz}, 1 \mathrm{~Hz}$ and $2 \mathrm{~Hz}$

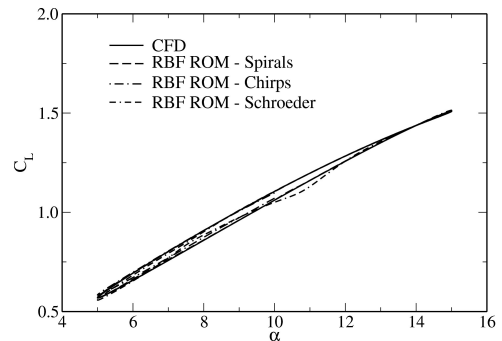

(a) $f=0.5 \mathrm{~Hz}$

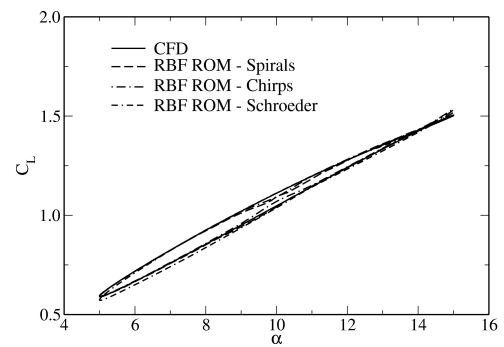

(b) $f=1 H z$

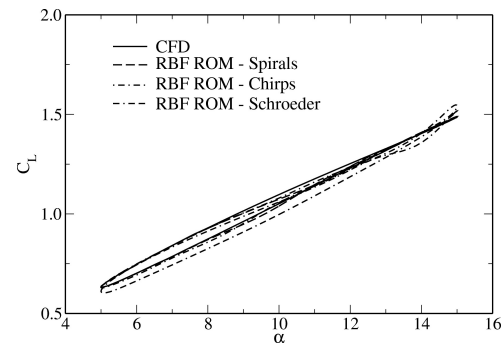

(c) $f=2 H z$

Figure 32. A comparison of SST and ROM predictions of lift coefficient for a dynamic pitching maneuver of $\alpha=10 \pm 5 \sin (\omega t)$ at frequencies of $f=0.5 \mathrm{~Hz}, 1 \mathrm{~Hz}$ and $2 \mathrm{~Hz}$

A comparison of ROM predictions of static lift and pitching moment to CFD data is shown in figure 35. As expected, in both cases it is the spiral ROM that gives the best results. It should be emphasized, that even if the comparison of the chirp based ROM and CFD is favorable, the results are extrapolated since the chirp training maneuver does not cover static regions of the regressor space. The Schroeder based ROM gives similar results as the chirp based ROM due to its poor coverage of the very low frequency regions. However, in this case, the static data is located within the regressor space coverage of the Schroeder training maneuver.

For this particular case, an extreme test of the ROM reliability and robustness is sinusoidal motion at $f=1.5 \mathrm{~Hz}$. This is because the pitching motion transitions from the state shown in figure 33(b) to the state 




(a) $f=0.5 \mathrm{~Hz}$



(b) $f=1 H z$



(c) $f=2 H z$

Figure 33. A comparison of SST and ROM predictions of pitching moment coefficient for a dynamic pitching maneuver with $\alpha=10 \pm 10 \sin (\omega t)$ at frequencies of $f=0.5 \mathrm{~Hz}, 1 \mathrm{~Hz}$ and $2 \mathrm{~Hz}$

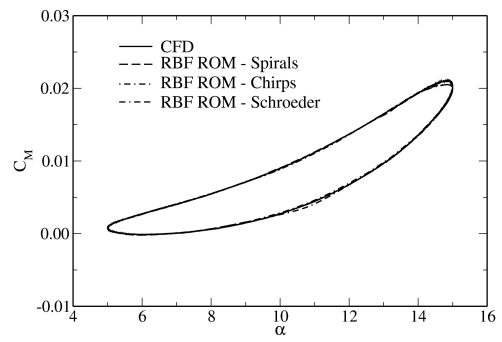

(a) $f=0.5 \mathrm{~Hz}$



(b) $f=1 H z$

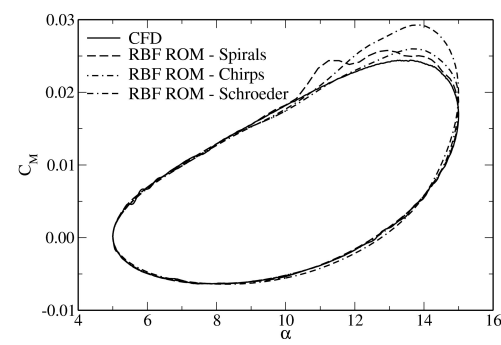

(c) $f=2 H z$

Figure 34. A comparison of SST and ROM predictions of pitching moment coefficient for a dynamic pitching maneuver of $\alpha=10 \pm 5 \sin (\omega t)$ at frequencies of $f=0.5 \mathrm{~Hz}, 1 \mathrm{~Hz}$ and $2 \mathrm{~Hz}$

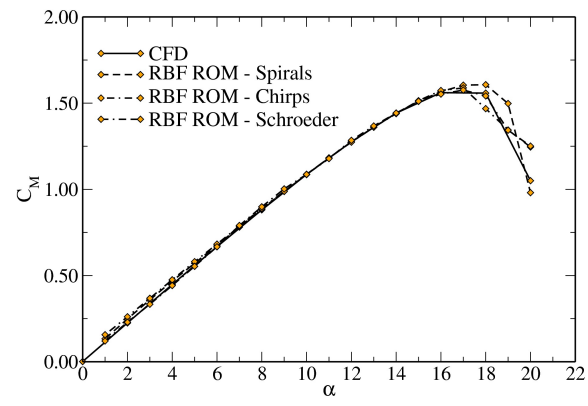

(a) $C_{L}$

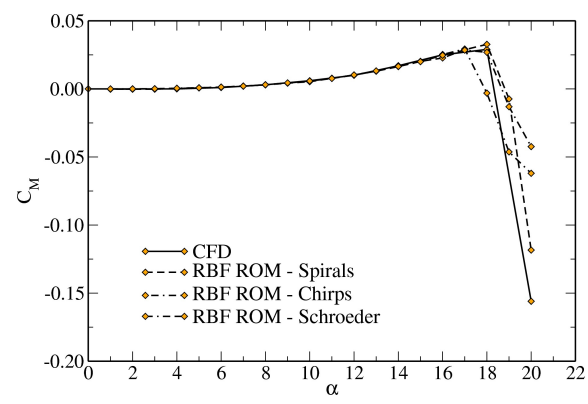

(b) $C_{M}$

Figure 35. A comparison of SST and ROM predictions of static lift and pitching moment coefficient for three different ROM that result from each type of training maneuver 
in figure 33(c). Sinusoidal CFD simulations were completed at $f=1.5 \mathrm{~Hz}$ for amplitudes of $A=10 \mathrm{deg}$, $9 \mathrm{deg}$ and 8deg, and predictions of lift and pitching moment coefficient are shown in figure 36. The CFD calculations indicate that the transition occurs between $f=1 \mathrm{~Hz}$ and $f=1.5 \mathrm{~Hz}$ since at $f=1.5 \mathrm{~Hz}$ the pitching moment curve has a shape similar to figure 33(c). Also shown in figure 36 is a comparison of the predicted aerodynamic loads for all three ROM. The chirp based and Schroeder based ROM predicted similar trends to the CFD solution, while the spiral based ROM predict a pitching moment curve similar to $f=1 \mathrm{~Hz}$, and therefore incorrectly predicted this transition in the range of $f=1.5 \mathrm{HZ}-2 \mathrm{~Hz}$.



(a) $A=10 \mathrm{deg}$

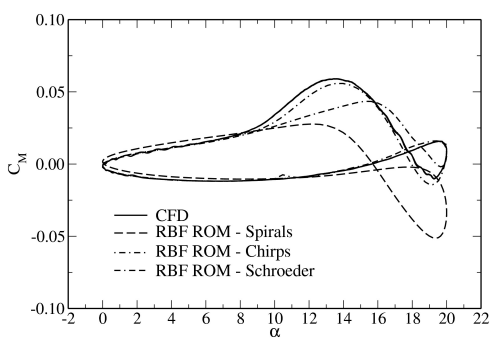

(d) $A=10 \mathrm{deg}$



(b) $A=9 d e g$

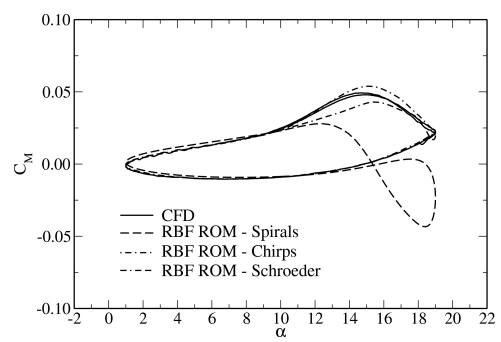

(e) $A=9 \mathrm{deg}$

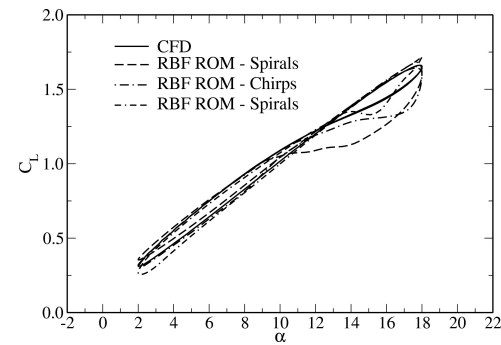

(c) $A=8 \mathrm{deg}$



(f) $A=8 d e g$

Figure 36. A comparison of SST and ROM predictions of lift and pitching moment coefficient for a dynamic pitching maneuver with $\alpha=10 \pm 1 A \sin (\omega t)$ at a frequency of $f=1.5 \mathrm{~Hz}$

\section{Plunging Motion}

The aerodynamic loads predicted by the four Fresnel chirp maneuvers previously presented were combined into one dataset and used as training data for a plunging ROM. Two ROMs were generated, the first model implemented $y, \dot{y}, \ddot{y}$ as the state variables while the second model implemented $\alpha_{e f f}, \dot{\alpha}_{e f f}, \ddot{\alpha}_{e f f}$. These models were then used to predict the lift and pitching moment for a sinusoidal oscillatory motion. As comparison of CFD and ROM predictions are shown in figure37. Comparing the ROM predictions with the CFD data, one can see that the ROM generated using $y, \dot{y}, \ddot{y}$ as the state variables predicts strong peaks in pitching moment which is not seen in the CFD data. The ROM generated using $\alpha_{e f f}, \dot{\alpha}_{e f f}, \ddot{\alpha}_{e f f}$ as the state variables resulted in fairly good agreement with CFD data. This is due to increased regular coverage of the regressor space defined in terms of $\alpha_{e f f}, \dot{\alpha}_{e f f}, \ddot{\alpha}_{e f f}$.

When plunging motion is defined by prescribing $\alpha_{e f f}$ the translation in the $y$-axis direction is determined by direct integration of the first derivative, $\dot{y}$. This integration introduces an integration constant that is dependent on the initial conditions. For most typical experimental and CFD applications this constant is arbitrary and therefore is often assumed to be one. However, for the current application this constant is important when determining the regressor space coverage and hence the applicability of a training maneuver.

As an example, consider the simple case of $\alpha_{e f f}=10 \sin (\omega t)$ at a frequency $f=0.85 \mathrm{~Hz}$. Using equation $6, \dot{y}=-v_{\infty} \tan \left(\alpha_{e f f}\right)$ and displacement is then given as follows

$$
y(t)=\int \dot{y} d t=-\int v_{\infty} \tan \left(\alpha_{e f f}\right) d t=f(t)+C,
$$

with an integration constant $C$. Using the initial condition $y(t)=0$ or $y(t) \approx-4.15$ for integration, locates the plunging motion at two different positions within the regressor space, as shown in figure 38(a). If using 




(a) $C_{L}$



(b) $C_{M}$

Figure 37. A comparison of SST and Fresnel chirp based ROM predictions of lift and pitching moment coefficient for a sinusoidal oscillatory motion of a plunging NACA0012 airfoil

CFD to predict the aerodynamic behavior, these two motions would produce identical results since the difference in position is not large enough to affect the Reynolds number. However, plotting the motions regressor space coverage together with the four Fresnel training maneuvers introduced previously, as in figure 38(b), shows that the motion defined using the initial condition $y(t)=0$ is almost entirely outside the training maneuvers regressor space coverage while the initial condition $y(0) \approx-4.15$ is almost entirely inside. Figure 39 then shows the ROM predictions of lift and pitching moment compared to CFD solutions for the motion defined with the initial condition $y(t)=0$ and $y(t) \approx-4.15$. The ROM based on the initial condition $y(t) \approx-4.15$ significantly improves the predictions of both lift and pitching moment.

The correct formulation of the initial condition for a specific maneuver of interest is defined by the training maneuver, which is an apparent paradox. In the case above, the initial portion of the $f=0.85 \mathrm{~Hz}$ sinusoidal oscillatory motion is replaced with the Fresnel chirp up to $f=0.85 \mathrm{~Hz}$. As which point the actual sinusoidal motion is analyzed. The resulting analyzed motion along with its position within the regressor space is shown in figure 40. Using this approach the analyzed motion is within the regressor space coverage of the Fresnel chirp training maneuvers.

It should be emphasized that such treatment is needed only needed when $y$-axis displacement is used as input to ROM. Using the $\alpha_{\text {eff }}$ defined regressor space avoids this paradox because it does not use position as a regressor variable.

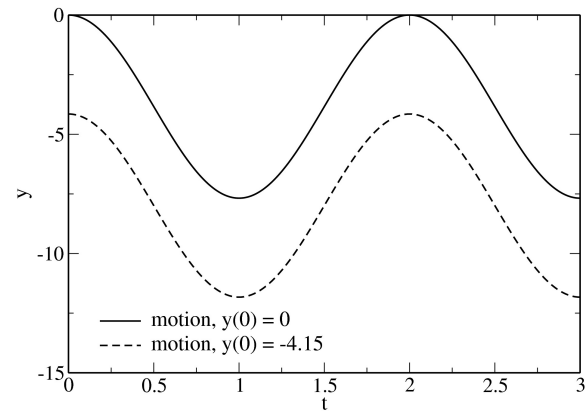

(a) $y-t$

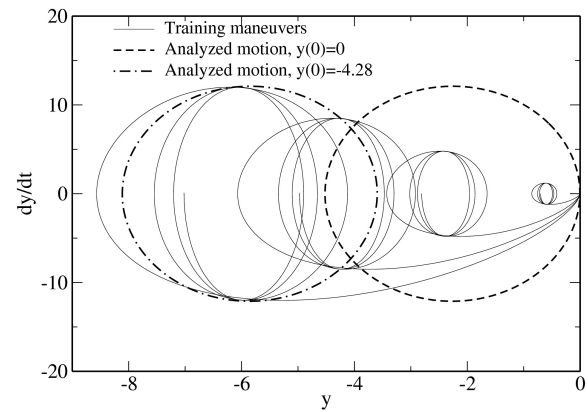

(b) $y-\dot{y}$

Figure 38. Regressor space coverage of two oscillatory motions with different values of the integration constant. Also shown is the regressor space coverage for the Fresnel chirp training maneuvers

\section{Extending regressor space coverage}

As seen in the results, for a reliable ROM, sufficient coverage of the regressor space is crucial. For the training maneuvers considered in the current study, altering maneuver properties such as amplitude result in changes in the regressor space coverage around a specific set of initial state variables. For the case of a two-dimensional pitching airfoil a single training maneuver results in regressor space coverage around one 




(a) $C_{L}$

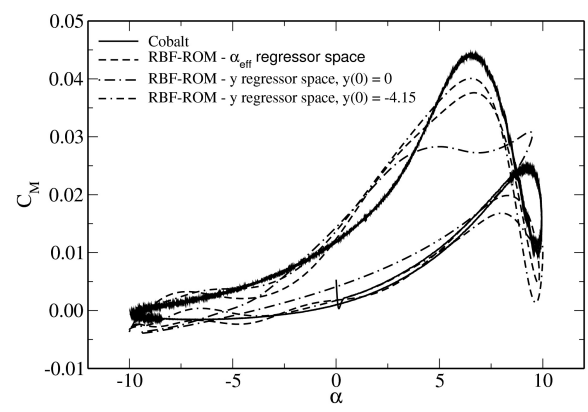

(b) $C_{M}$

Figure 39. Lift and pitching moment coefficient, sinusoidal motion, $y$ and $\alpha_{e f f}$ - regressor space

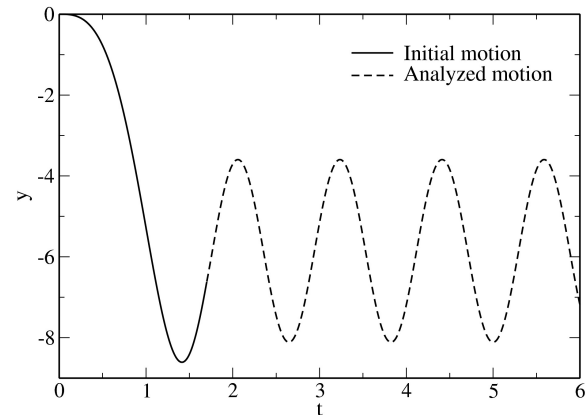

(a) $y-t$

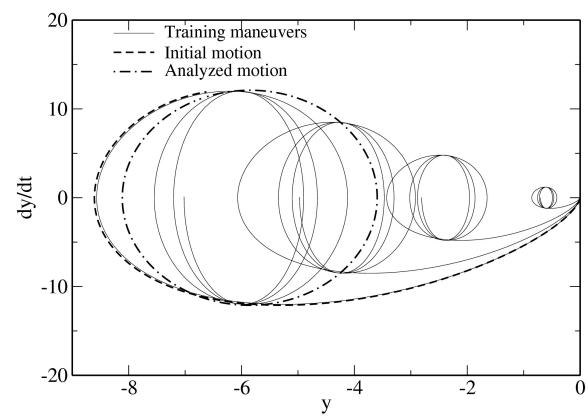

(b) $y-\dot{y}$

Figure 40. Procedure for correctly positioning a motion of interest within the training maneuver regressor space coverage when the maneuvers are defined in terms of $y$-axis displacement 
initial angle of attack, $\alpha_{0}$. However, certain properties of the regressor space coverage remain unchanged. For example, the acceleration is always negative for the downward stroke of the pitching airfoil and positive for the upward stroke. The only exception is the Schroeder maneuver where the first and third quadrants of the acceleration plane is to a limited extent covered. To extend the coverage of the considered maneuvers, it is necessary to alter the initial angle of attack, $\alpha_{0}$, about which the maneuvers are build. This effectively traverses the regressor space coverage along the primary variable axis. Figure 41 shows such an example for the chirp training maneuver.



Figure 41. Extended regressor space covered by multiple chirp maneuvers with varying $\alpha_{0}$

\section{Conclusion}

Improved methodologies for implementing computational fluid dynamics in full aircraft stability and control analysis were further developed using a two-dimensional NACA0012 airfoil. The premise being that any improvements made for a two-dimensional airfoil will also be valid for full aircraft configurations. Several training maneuvers that improve the regressor map coverage along with a new approach to reduced order model development were investigated.

For aircraft S\&C applications, the ability to cover the relevant regressor space and to capture a broad spectrum of physical phenomena within that regressor space was shown to be crucial. This is different from earlier philosophies that primarily focused on frequency coverage. Of all the investigated maneuvers, spiral maneuvers augmented with a piece wise linear spiral maneuver resulted in the best regressor space coverage for static and very low frequency data, however, they are the most computationally demanding. Chirp maneuvers generally resulted in the best regressor space coverage at higher frequencies. The Schroeder maneuver resulted in coverage somewhere between the previous two, but had the additional advantage of covering the first and third quadrant of the $\alpha-\ddot{\alpha}$ regressor space, which is left uncovered by spiral and chirp maneuvers. On the other hand, the regressor space coverage by the Schroeder maneuver is somewhat irregular and difficult to control systematically.

The aerodynamic loads resulting from the various CFD training maneuvers were then used to generate reduced order models, which were then used to predict lift and pitching moment for static conditions and several dynamic sinusoidal maneuvers. The predicted aerodynamic loads were compared to CFD validation data. The reduced order model implemented was based on radial basis functions, and showed improvements over previous regression based models. This was primarily in the ability to predict both linear and nonlinear aerodynamic behavior with a single aerodynamic loads model, a task that has been difficult to achieve in the past. All of the reduced order models resulted in reliable predictions in both the linear and nonlinear aerodynamic regions. Apart from static and very low frequency data, the chirp maneuvers resulted in the most robust and reliable reduced order models. This was, in part, due to the very regular coverage of the regressor space by this maneuver. However, for reliable predictions of very low frequency and even static aerodynamic behavior, which is where the most extreme nonlinear aerodynamic behavior occurs, the spiral based reduced order models were superior.

This article highlights that a well designed training maneuver can provide enough high quality training data for a reduced order model to predict strong nonlinearities located within the covered regressor space. 


\section{Acknowledgments}

The authors would like to thank the Air Force Office of Scientific Research, AFOSR, the United States Air Force Academy, USAFA - Modeling and Simulation Research Center, the National Research Council (NRC) and the United States Air Force SEEK Eagle Office for their generous financial support throughout this project. The Arctic Region Supercomputing Center is acknowledged for providing access to high performance computing facilities.

\section{References}

${ }^{1}$ Chambers, J. R. and Hall, R. M., "Historical Review of Uncommanded Lateral-Directional Motions at Transonic Conditions," Journal of Aircraft, Vol. 41, No. 3, May-June 2004, pp. 436-447.

${ }^{2}$ Hall, R. M., Woodson, S. H., and Chambers, J. R., "Accomplishments of the Abrupt-Wing-Stall Program," Journal of Aircraft, Vol. 42, No. 3, May-June 2005, pp. 653-660.

${ }^{3}$ Bowers, A. H., Pahle, J. W., Wilson, R. J., Flick, B. C., and Rood, R. L., "An Overview of the NASA F- 18 High Alpha Research Vehicle," NASA TM-4772, Oct. 1996.

${ }^{4}$ Wang, K. C. and Iliff, K. W., "Retrospective and Recent Examples of Aircraft Parameter Identification at NASA Dryden Flight Research Center," Journal of Aircraft, Vol. 41, No. 4, July-August 2004, pp. 752-764.

${ }^{5}$ Moses, R. W., "Fin buffeting features of an early F-22 model," AIAA Paper 2000-1695, April 2000.

${ }^{6}$ Potoczsky, A. S. and Moses, R. W., "An analysis Method to Predict Tail Buffet Loads of Fighter Aircraft," AIAA Paper 2005-2291, April 2005.

${ }^{7}$ Murman, M. M., Chaderjian, N. M., and Pandya, S. A., "Automation of a Navier-Stokes S\&C database generation for the Harrier in ground effect," AIAA Paper 2002-259, 2002.

${ }^{8}$ Chaderjian, N. M., Ahmad, J., Pandya, S., and Murman, S., "Progress Toward Generation of a Navier-Stokes Database for a Harrier in Ground Effect," AIAA Paper 2002-5966, 2002.

${ }^{9}$ Rogers, S. E., Aftomis, M. J., Pandya, S. A., Chaderjian, N. M., Tejnil, E. T., and Ahmad, J. U., "Automated CFD Parameter Studies on Distributed Parallel Computers," AIAA Paper 2003-4229, June 2003.

${ }^{10}$ McDaniel, D. R., Cummings, R. M., Bergeron, K., Morton, S. M., and Dean, J. P., "Comparisons of CFD Solutions of Static and Maneuvering Fighter Aircraft with Flight Test Data," 3rd International Symposium on Integrating CFD and Experiments in Aerodynamics, 20-21 June 2007, US Air Force Academy, CO.

${ }^{11}$ Matthews, L. L. and Schwartz, M. S., "Modeling and Simulations of a Dynamic Maneuvering F-16," AIAA Student Conference, May, 2008.

${ }^{12}$ Morelli, E. A., "System IDentification Programs for AirCraft (SIDPAC)," AIAA Paper 2002-4704, 2002.

${ }^{13}$ Jeans, T., McDaniel, D., Cummings, R., and Bergeron, K., "Lower-Order Aerodynamic Loads Modeling of a Maneuvering Generic Fighter Using DDES Simulations," AIAA-2009-0094, 47th AIAA Aerospace Sciences Meeting including The New Horizons Forum and Aerospace Exposition, Orlando, Florida, Jan. 5-8, 2009.

${ }^{14}$ Görtz, S., McDaniel, D., and Morton, S., "Towards an Efficient Aircraft Stability and Control Analysis Capability Using High-Fidelity CFD," AIAA-2007-1053, 45th AIAA Aerospace Sciences Meeting and Exhibit, Reno, Nevada, Jan. 8-11, 2007.

${ }^{15}$ Forsythe, J. R. and Woodson, S. H., "Unsteady Computations of Abrupt Wing Stall Using Detached-Eddy Simulation," Journal of Aircraft, Vol. 42, No. 3, May-June 2005, pp. 606-616.

${ }^{16}$ Morton, S. A., Forsythe, J. R., Mitchell, A. M., and Hajek, D., "Detached-Eddy Simulations and Reynolds-Averaged Navier-Stokes Simulations of Delta Wing Vortical Flowfields," Journal of Fluids Engineering, Vol. 124, No. 4, 2002 , pp. $924-932$.

${ }^{17}$ Forsythe, R., Squires, K. D., Wurtzler, K. E., and Spalart, P. R., "Detached-Eddy Simulation of Fighter Aircraft at High Alpha," Journal of Aircraft, Vol. 41, No. 2, March-April 2004, pp. 193-200.

${ }^{18}$ Forsythe, R., Fremaux, C. M., and Hall, R. M., "Calculation of Static and Dynamic Stability Derivatives of the F/A-18E in Abrupt Wing Stall Using RANS and DES," in Proceedings of International Conference for CFD, Toronto, Canada, 2004.

${ }^{19}$ Morton, A., Forsythe, J. R., Squires, K. D., and Cummings, R. M., "Detached-Eddy Simulations of Full Aircraft Experiencing Massively Separated Flows," Computational Fluid Dynamics Journal ISCFD Japan, Vol. 13, No. 3, Jan. 2005.

${ }^{20}$ Jeans, T., McDaniel, D., Cummings, R., and Mason, W., "Aerodynamic Analysis of a Generic Fighter Using Delayed Detached-Eddy Simulation," Journal of Aircraft, Vol. 46, No. 4, July-August 2009, pp. 1326-1339.

${ }^{21}$ Jirásek, A. and Cummings, R. M., "Application of Volterra Functions to X-31 Aircraft Model Motion," AIAA 2009-3629, 27th AIAA Applied Aerodynamics Conference, San Antonio, Texas, June 22-25, 2009.

${ }^{22}$ O'Neill, C. and Arena, A., "Time Domain Training Signals Comparison for Computational Fluid Dynamics Based Aerodynamic Identification," Journal of Aircraft, Vol. 42, No. 2, Jan. 2005, pp. 421-428.

${ }^{23}$ Murpy, P. C. and Klein, V., "Validation of Methodology for Estimating Aircraft Unsteady Aerodynamics Parameters from Dynamic Wind Tunnel Tests," AIAA Paper 2003-5397, AIAA Athmospheric Flight Mechanics Conferencer and Exhibit, August 11-14, Austin TX, 2003.

${ }^{24}$ Morelli, E. A., "Real Time Parameters Estimation in the Frequency Domain," AIAA-99-4043, 1999.

${ }^{25}$ Tobak, M., Chapman, G. T., and Ünal, A., "Modeling Aerodynamic Discontinuities and the Onset of Chaos in Flight Dynamical Systems," NASA Technical Memorandum 89420, Dec. 1986.

${ }^{26}$ Silva, W. A., "Identification of Nonlinear Aeroelastic Systems based of the Volterra Theory: Progress and Opportunities," Nonlinear Dynamics, Springer, Vol. 39, No. 9, Sept. 2005.

${ }^{27}$ Beran, P. S., Lucia, D. J., and Pettit, C. L., "Reduced-order modelling of limit-cycle oscillation for aeroelastic systems," Journal of Fluids and Structures, Vol. 19, Sept. 2004. 
${ }^{28}$ Schroeder, M. R., "Synthesis of Low-Peak Factor Signals and Binary Sequences with Low Autocorrelation," IEEE Transcriptions of Information Technology, Vol. 16, 1970, pp. 85-89.

${ }^{29}$ Strang, W. Z., Tomaro, R. F., and Grismer, M. J., "The Defining Methods of Cobalt60: A Parallel, Implicit, Unstructured Euler/Navier Stokes Flow Solver," AIAA Paper 99-0786, 37th AIAA Aerospace Sciences Meeting and Exhibit, Jan. 1999.

${ }^{30}$ Gottlieb, J. J. and Groth, C. P. T., "Assessment of Riemann Solvers for Unsteady One-Dimensional Inviscid Flows of Perfect Gasses," Journal of Computation Physics, Vol. 78, No. 2, Jan. 1998, pp. 437-458.

${ }^{31}$ Tomaro, R. F., Strang, W. Z., and Sankar, L. N., "An implicit algorithm for solving time dependent flows on unstructured grids," AIAA Paper 1997-0333, Jan. 1997.

32 "Cobalt V4.0, www.cobaltcfd.com," Tech. rep., June 2008.

${ }^{33}$ Menter, F. R., "Two-equation Eddy Viscosity Turbulence Models For Engineering Application," AIAA Journal, Vol. 32, No. 8, Aug. 1994, pp. 1598-1605.

${ }^{34}$ Abbot, I. H. and Von Doenhoff, A. E., Theory of Wing Sections, Including a Summary of Airfoil Data, AIAA Education Series, Dover Publications Inc., New York, 1959.

35 "Solid Mesh 5.45 3D Users Manual," Mississippi State University Computational Simulation and Design Center, July 2008.

${ }^{36}$ Amoignon, O., "AESOP - A Numerical Platform for Aerodynamic Shape Optimization," Optimization and Engineering, Springer Netherlands, Jan. 2009.

${ }^{37}$ Rendall, T. and Allen, C., "Unified CFD-CSD Interpolation and Mesh Motion using Radial Basis Function," International Journal for Numerical Methods in Engineering, Vol. 74, No. 10, 2008, pp. 1519-1559.

${ }^{38}$ Morris, A. M., Allen, C. B., and Rendall, T. C. S., "Aerodynamic Shape Optimization Applied to Modern Transport Wing," AIAA Journal, Vol. 47, No. 7, July 2009, pp. 1647-1659.

${ }^{39}$ Krishnamurthy, T., "Response Surface Approximation with Augmented and Compactly Supported Radial Basis Functions," AIAA 2003-1748, 44th AIAA/ASME/ASCE/AHS Structures, Structural Dynamics, and Material Conference, 7-10 April, Norfolk, VA, 2003

${ }^{40}$ Hardy, R. L., "Multiquadratic Equations of Topography and Other Irregular Surfaces," Journal of Geophysical Research, Vol. 76, 1971, pp. 1905-1915.

${ }^{41}$ Jeng, Y.-N., Huang, P. G., and Cheng, Y.-C., "Decomposition of one-dimensional waveform using iterative Gaussian diffusive filtering methods," Proceedings of the Royal Society A: Mathematical, Physical and Engineering Sciences 464, March, pp. 1673-1695. 